\title{
OPTIMAL CONTROL OF STATIONARY, LOW MACH NUMBER, HIGHLY NONISOTHERMAL, VISCOUS FLOWS *
}

\author{
Max D. GunzBurger ${ }^{1}$ And O.Yu. Imanuvilov ${ }^{1}$
}

\begin{abstract}
An optimal control problem for a model for stationary, low Mach number, highly nonisothermal, viscous flows is considered. The control problem involves the minimization of a measure of the distance between the velocity field and a given target velocity field. The existence of solutions of a boundary value problem for the model equations is established as is the existence of solutions of the optimal control problem. Then, a derivation of an optimality system, i.e., a boundary value problem from which the optimal control and state may be determined, is given.
\end{abstract}

Résumé. Dans cet article, on considère un problème de contrôle optimal pour un fluide visqueux, stationnaire et fortement non-isothermique, à petit nombre de Mach. On regarde d'abord un problème de minimisation pour l'écart entre le champ de vitesse et un champ de vitesse donné. On montre l'existence de solutions pour ce problème ainsi que pour le problème de contrôle optimal initial. On donne ensuite un système vérifié par les solutions du probléme de contrôle optimal, système qui consiste en un problème aux limites dont on peut obtenir le contrôle optimal et l'état.

AMS Subject Classification. 49J20, 49K20, 76N99.

Received January 31, 2000.

\section{INTRODUCTION}

In recent times, there has been many studies, both of a mathematical and an engineering nature, of control and optimization problems for the Navier-Stokes system for incompressible, viscous flows. There have also been a number computational studies of such problems for compressible flows, including flows with shock waves and other discontinuities. Here we are concerened with the mathematical analysis of control and optimization problems for a simplified yet useful model for compressible flows that is valid for low Mach number, highly nonisothermal, viscous flows. One important application to which this model applies is flows in chemical vapor deposition reactors; see, e.g. $[1,3,6]$.

\footnotetext{
Keywords and phrases: Optimal control, compressible flows, viscous flows, low Mach number flows.

* The work of MDG was supported in part by the U.S. Air Force Office of Scientific Research under grant number F49620-95$1-040 \%$.

1 Department of Mathematics, Iowa State University, Ames IA 50011-2064, U.S.A.;

e-mail: gunzburg@iastate.edu \& vika@iastate.edu
} 
Specifically, given the bounded, two-dimensional domain $\Omega$ with boundary $\Gamma$, the control problem we consider is in the context of the boundary value problem

$$
\begin{aligned}
-\Delta \mathbf{u}+\rho \mathbf{u} \cdot \nabla \mathbf{u}+\nabla p+\beta\left(\begin{array}{c}
0 \\
1
\end{array}\right) \rho T=\mathbf{f} \quad \text { in } \Omega, \\
\nabla \cdot(\rho \mathbf{u})=0 \quad \text { in } \Omega, \\
\left.\mathbf{u}\right|_{\Gamma}=\mathbf{0}, \\
-\Delta T+\rho \mathbf{u} \cdot \nabla T=q \quad \text { in } \Omega, \\
\rho(1+T)=1 \quad \text { in } \Omega,
\end{aligned}
$$

and

$$
\left.T\right|_{\Gamma}=\theta,
$$

where $\mathbf{u}, p, \rho$, and $T$ denote the (appropriately nondimensionalized) velocity, pressure, density, and temperature fields, respectively. In a boundary value problem, the functions $q, \mathbf{f}$, and $\theta$ are given as is the constant $\beta=1 / F r$, where $\mathrm{Fr}$ denotes the Froude number. In the control problem we will consider, the function $\theta$ will act as a control and is therefore unknown. We assume, for the boundary value problem, that

$$
q \geq 0 \quad \text { in } \Omega
$$

and

$$
\theta \geq 0 \quad \text { on } \Gamma
$$

the second of these will also be a requirement of candidate optimal controls in the optimization problem we will consider. In the nondimensional model (1-8), the variables and data functions have been appropriately rescaled. Also, $T$ actually denotes a temperature difference with respect to a reference temperature.

A detailed derivation of the model (1-8) is given in [6]; see also [4]. Here, we only mention that the model is derived from the equations for viscous, compressible flows (see, e.g. [7]) under the assumptions that the Mach number $M=V_{0} / a_{0}$ and the hydrostatic compressibility $g L_{0} /\left(R T_{0}\right)$ is small, where $g$ is the constant gravitational acceleration, $a_{0}$ is the speed of sound, $R$ is the gas constant, and $L_{0}, V_{0}$, and $T_{0}$ are length, velocity, and temperature scales, respectively. Specifically, it is assumed that $M^{2} \ll 1$ and $g L /\left(R T_{0}\right) \ll 1$ in such a way that $g L /\left(M^{2} R T_{0}\right)=O(1)$. This model is not useful for describing acoustic phenomena due to the independence of the pressure from the density and temperature; see (5) which is the simplified ideal gas equation of state valid in the limits given above.

The essential differences between the model (1-8) and the Navier-Stokes system are evident. Not only is there coupling between the conservation of energy equation equation (4) and the conservation of momentum and mass equations (1) and (2), respectively, but we also have the appearance of the density function $\rho$. Note, in particular, that from (2), the velocity field $\mathbf{u}$ itself is not solenoidal.

The specific control problem we consider is to find a boundary temperature distribution $\theta$ and a velocity field $\mathbf{u}$ such that the functional

$$
J(\mathbf{u}, \theta)=\frac{1}{2} \int_{\Omega}\left|\mathbf{u}-\mathbf{u}_{0}\right|^{2} d \Omega+\frac{1}{2} \int_{\Gamma}\left(\left|\theta-\theta_{0}\right|^{2}+\left|\frac{\partial\left(\theta-\theta_{0}\right)}{\partial \vec{\tau}}\right|^{2}\right) d \Gamma,
$$


is minimized subject to the system (1-8) being satisfied. Here, $\mathbf{u}_{0}$ and $\theta_{0}$ are given functions and $\vec{\tau}=\vec{\tau}(x)$ denotes the unit, continuous tangent vector to $\Gamma$. The first term in the functional $J(\cdot, \cdot)$ measures the distance between the velocity field $\mathbf{u}$ and a prescribed velocity field $\mathbf{u}_{0}$. The second term is used to limit the size of the control function $\theta$.

The plan of the rest of the paper is as follows. In Section 2, we study the existence of solutions of the boundary value problem, i.e., of the system (1-8) where $\theta$ is a prescribed function. In Section 3, we study the existence of solutions of the optimal control problem, i.e., the problem of finding $\theta$ and $\mathbf{u}$ such that the functional of (9) is minimized subject to (1-8). Then, in Section 4, we derive an optimality system, i.e., a boundary value problem from which the optimal control $\theta$ and optimal state $(\mathbf{u}, T, p)$ may be determined.

\section{The Boundary VAlue PRoblem}

Let $\Omega$ denote a bounded, open set of $\mathbb{R}^{2}$ with boundary $\Gamma \in C^{2}$. We define the function spaces

$$
\begin{gathered}
\mathbf{H}=\left\{\mathbf{v} \in\left[L^{2}(\Omega)\right]^{2} \quad|\quad \nabla \cdot \mathbf{v}=0, \quad(\mathbf{v} \cdot \mathbf{n})|_{\Gamma}=0\right\}, \\
\mathbf{V}=\left\{\mathbf{v} \in\left[W_{2}^{1}(\Omega)\right]^{2} \quad|\quad \nabla \cdot \mathbf{v}=0, \quad \mathbf{v}|_{\Gamma}=\mathbf{0}\right\}, \\
\widetilde{L}^{2}(\Omega)=\left\{p \in L^{2}(\Omega) \quad \mid \int_{\Omega} p d \Omega=0\right\}, \\
\mathbf{H}^{s}=\mathbf{V} \cap\left[W_{2}^{s}(\Omega)\right]^{2}, \quad s \geq 1, \quad \text { and } \quad \widetilde{W}_{2}^{s}(\Omega)=W_{2}^{s}(\Omega) \cap \widetilde{L}^{2}(\Omega), \quad s \geq 0,
\end{gathered}
$$

where $\mathbf{n}$ denotes the unit outer normal along $\Gamma$. We have the following result concerning the existence of solutions of the boundary value problem (1-6) for which $\theta$ as well as $\mathbf{f}$ and $q$ are prescribed functions.

Theorem 2.1. Let $\theta \in W_{2}^{s}(\Gamma), s \in\left[1, \frac{3}{2}\right], \mathbf{f} \in\left[L^{2}(\Omega)\right]^{2}, q \in L^{2}(\Omega)$, and let the conditions (7) and (8) hold true. Then, the problem (1-6) has at least one solution $(\mathbf{u}, T, p) \in\left[W_{2}^{s+\frac{1}{2}}(\Omega)\right]^{2} \times W_{2}^{s+\frac{1}{2}}(\Omega) \times \widetilde{L}^{2}(\Omega)$ with $\rho \mathbf{u} \in \mathbf{H}^{2}$. Moreover, if $(\mathbf{u}, T, p) \in\left[W_{2}^{1}(\Omega)\right]^{2} \times W_{2}^{\frac{3}{2}}(\Omega) \times \widetilde{L}^{2}(\Omega)$ is a solution of $(1)-(6)$, then it belongs to the space $\left[W_{2}^{s+\frac{1}{2}}(\Omega)\right]^{2} \times W_{2}^{s+\frac{1}{2}}(\Omega) \times \widetilde{L}^{2}(\Omega)$ and there exists a continuous function $M(t)$ such that every solution to this problem from the space $\left[W_{2}^{1}(\Omega)\right]^{2} \times W_{2}^{\frac{3}{2}}(\Omega) \times \widetilde{L}^{2}(\Omega)$ satisfies the a priori estimate

$$
\|(\mathbf{u}, T, p)\|_{\left[W_{2}^{s+\frac{1}{2}}(\Omega)\right]^{2} \times W_{2}^{s+\frac{1}{2}}(\Omega) \times \widetilde{L}^{2}(\Omega)} \leq M\left(\|\theta\|_{W_{2}^{s}(\Gamma)}+\|q\|_{L^{2}(\Omega)}+\|\mathbf{f}\|_{\left[L^{2}(\Omega)\right]^{2}}\right) .
$$

To prove Theorem 2.1, we need some preliminary results, the first of which is a regularity result for solutions of the generalized Stokes system.

Proposition 2.2. For any $\mathbf{f} \in\left[L^{2}(\Omega)\right]^{2}$ and $g \in \widetilde{W}_{2}^{\ell}(\Omega), \ell \in[0,1]$, there exist a solution $(\mathbf{u}, p) \in\left[W_{2}^{\ell+1}(\Omega)\right]^{2} \times$ $\widetilde{W}_{2}^{\ell}(\Omega)$ to the generalized Stokes problem

$$
\begin{gathered}
-\Delta \mathbf{v}=\nabla p+\mathbf{f} \quad \text { in } \Omega \\
\nabla \cdot \mathbf{v}=g \quad \text { in } \Omega, \quad \text { and }\left.\quad \mathbf{v}\right|_{\Gamma}=\mathbf{0} .
\end{gathered}
$$


Moreover, this solution is unique in the space $\left[W_{2}^{1}(\Omega)\right]^{2} \times \widetilde{L}^{2}(\Omega)$ and satisfies the estimate

$$
\|\mathbf{v}\|_{\left[W_{2}^{\ell+1}(\Omega)\right]^{2}}+\|p\|_{W_{2}^{\ell}(\Omega)} \leq C_{1}\left(\|\mathbf{f}\|_{\left[L^{2}(\Omega)\right]^{2}}+\|g\|_{W_{2}^{\ell}(\Omega)}\right) .
$$

Proof. For $\ell=0$ or 1, the statement of this proposition proved in [8] (Prop. 2.2, p. 33, Th. 2.4, p. 31). Thus, we need only consider the case when $\ell \in(0,1)$. Let $T$ be the linear operator on $\left[L^{2}(\Omega)\right]^{2} \times \widetilde{L}^{2}(\Omega)$ such that $T(\mathbf{f}, g)=(\mathbf{v}, p)$, where the pair $(\mathbf{v}, p)$ is the solution to the generalized Stokes system. Obviously, $T \in \mathcal{L}\left(\left[L^{2}(\Omega)\right]^{2} \times \widetilde{L}^{2}(\Omega) ;\left[W_{2}^{1}(\Omega)\right]^{2} \times \widetilde{L}^{2}(\Omega)\right) \cap \mathcal{L}\left(\left[L^{2}(\Omega)\right]^{2} \times \widetilde{W}_{2}^{1}(\Omega) ;\left[W_{2}^{2}(\Omega)\right]^{2} \times \widetilde{W}_{2}^{1}(\Omega)\right)$. Since by definition (see [5], p. 40), $W_{2}^{\ell}(\Omega)=\left[W_{2}^{1}(\Omega), L^{2}(\Omega)\right]_{\xi},(1-\xi)=\ell$, the theorem on the interpolation of quotient spaces (see [5], Th. 13.2, p. 90) implies that $\widetilde{W}_{2}^{\ell}(\Omega)=\left[W_{2}^{1}(\Omega) \cap \widetilde{L}^{2}(\Omega), \widetilde{L}^{2}(\Omega)\right]_{\xi},(1-\xi)=\ell$. Thus, by [5] (Th. 5.1, p. 27),

$$
T \in \mathcal{L}\left(\left[L^{2}(\Omega)\right]^{2} \times \widetilde{W}_{2}^{\ell}(\Omega) ;\left[W_{2}^{1+\ell}(\Omega)\right]^{2} \times \widetilde{W}_{2}^{\ell}(\Omega)\right)
$$

For the next preliminary result, we introduce, for an arbitrary function $\mathbf{v} \in \mathbf{H} \cap\left[L^{5}(\Omega)\right]^{2}$, the mapping $\phi: \mathbf{v} \rightarrow$ $\phi(\mathbf{v})$, where $\phi(\mathbf{v})$ is the solution to the boundary value problem

$$
L \phi=-\Delta \phi+\mathbf{v} \cdot \nabla \phi=q \quad \text { in } \Omega \quad \text { and }\left.\quad \phi\right|_{\Gamma}=\theta,
$$

where $q \in L^{2}(\Omega)$ and $\theta \in W_{2}^{1}(\Gamma)$ satisfy to (7) and (8). $\phi(\mathbf{v})$ of (11) from the space $L^{2}(\Omega)$ in the following sense:

$$
\left(\phi(\mathbf{v}), L^{*} \psi\right)_{L^{2}(\Omega)}=(q, \psi)_{L^{2}(\Omega)}+\left(\theta, \frac{\partial \psi}{\partial \nu}\right)_{L^{2}(\Gamma)} \quad \forall \psi \in W_{2}^{2}(\Omega) \cap \stackrel{\circ}{W_{2}^{1}}(\Omega),
$$

where formally, since $\nabla \cdot \mathbf{v}=0$, the adjoint operator $L^{*}$ has the form

$$
L^{*} \psi=-\Delta \psi-\mathbf{v} \cdot \nabla \psi
$$

For the problem (11), we have the following result which includes a priori estimates for $\phi(\mathbf{v})$ which are independent of $\mathbf{v}$. Such estimates will be needed later to apply a fixed point theorem.

Proposition 2.3. The solution of the problem (11) for the mapping $\phi: \mathbf{H} \cap\left[L^{5}(\Omega)\right]^{2} \rightarrow L^{2}(\Omega)$ exists and is unique. Moreover, we have that

$$
\begin{gathered}
\phi(\mathbf{v}) \geq 0 \quad \text { in } \Omega, \\
\|\phi(\mathbf{v})\|_{L^{k}(\Omega)} \leq C_{2}(k)\left(\|q\|_{L^{2}(\Omega)}+\|\theta\|_{W_{2}^{1}(\Gamma)}\right) \quad \forall k \in \mathbb{N}_{+},
\end{gathered}
$$

and

$$
\|\phi(\mathbf{v})\|_{W_{2}^{\frac{1}{2}+s}(\Omega)} \leq C_{3}\left(\|\mathbf{v}\|_{\left[L^{5}(\Omega)\right]^{2}}\right)\left(\|q\|_{L^{2}(\Omega)}+\|\theta\|_{W_{2}^{1}(\Gamma)}\right), \quad s \in(1,3 / 2],
$$

where $C_{2}(k)$ is independent of $\mathbf{v}, q, \theta$ and $C_{3}$ is independent of $q, \theta$ and depends continuously on $\|\mathbf{v}\|_{\left[L^{5}(\Omega)\right]^{2}}$.

Proof. To prove the existence of a solution to (11), we assume $\mathbf{v} \in \mathbf{V} \cap\left[W_{2}^{\frac{3}{2}}(\Omega)\right]^{2}$ and rewrite the problem in the abstract form

$$
P \phi(\mathbf{v})=\left(P_{1}+P_{2}\right) \phi(\mathbf{v})=(q, \theta),
$$


where $P_{1} y=\left(-\Delta y,\left.y\right|_{\Gamma}\right)$ and $P_{2} y=(\mathbf{v} \cdot \nabla y, 0)$. We now show that the operator $P: W_{2}^{s+\frac{1}{2}}(\Omega) \rightarrow W_{2}^{s-\frac{3}{2}}(\Omega) \times$ $W_{2}^{s}(\Gamma)$ has the property

$$
\operatorname{Im} P=W_{2}^{s-\frac{3}{2}}(\Omega) \times W_{2}^{s}(\Gamma)
$$

It is well known (see [5]) that (15) holds true for the operator $P_{1}$, i.e.,

$$
\operatorname{Im} P_{1}=W_{2}^{s-\frac{3}{2}}(\Omega) \times W_{2}^{s}(\Gamma)
$$

Moreover, due to the inequality

$$
\|\mathbf{v} \cdot \nabla \phi\|_{L^{2}(\Omega)} \leq C_{1}\|\mathbf{v}\|_{\left[L^{5}(\Omega)\right]^{2}}\|\phi\|_{W_{2}^{\frac{3}{2}}(\Omega)}
$$

the operator $P_{2}: W_{2}^{s+\frac{1}{2}}(\Omega) \rightarrow W_{2}^{s-\frac{3}{2}}(\Omega) \times W_{2}^{s}(\Gamma)$ is compact. Hence, instead of (15), it suffices to prove

$$
\overline{\operatorname{Im} P}=W_{2}^{s-\frac{3}{2}}(\Omega) \times W_{2}^{s}(\Gamma)
$$

On the other hand (16) implies that (17) is equivalent to the following: for the operator $L: W_{2}^{s+\frac{1}{2}}(\Omega) \cap \stackrel{\circ}{W_{2}^{1}}(\Omega) \rightarrow$ $W_{2}^{s-\frac{3}{2}}(\Omega)$

$$
\overline{\operatorname{Im} L}=W_{2}^{s-\frac{3}{2}}(\Omega)
$$

Suppose that (18) does not hold true. In that case, there exists a nonzero solution $z \in L^{2}(\Omega)$ to the boundary value problem

$$
L^{*} z=0 \quad \text { in } \Omega \quad \text { and }\left.\quad z\right|_{\Gamma}=0
$$

Taking into account the fact that $\mathbf{v} \cdot \nabla z \in W_{2}^{-1}(\Omega)$, we obtain from (19) that $z \in W_{2}^{1}(\Omega)$. This regularity implies immediately $\mathbf{v} \cdot \nabla z \in L^{2}(\Omega)$. Hence, $z \in W_{2}^{2}(\Omega)$ and taking the scalar product of this function with (19), we obtain $\int_{\Omega}|\nabla z|^{2} d \Omega=0$. This, together with the boundary condition in (19), implies that $z \equiv 0$ which is a contradiction.

Now, let $\mathbf{v} \in \mathbf{H} \cap\left[L^{5}(\Omega)\right]^{2}$ be an arbitrary function and the sequence $\mathbf{v}_{i} \in \mathbf{V} \cap\left[C^{2}(\bar{\Omega})\right]^{2}$ be its approximation in the space $\mathbf{H} \cap\left[L^{5}(\Omega)\right]^{2}$ so that

$$
\mathbf{v}_{i} \rightarrow \mathbf{v} \quad \text { in } \mathbf{H} \cap\left(L^{5}(\Omega)\right)^{2} \quad \text { as } i \rightarrow+\infty
$$

Above we proved that for each $\mathbf{v}_{i}$ there exists a solution $y_{i} \in W_{2}^{s+\frac{1}{2}}(\Omega)$ to the boundary problem

$$
L_{i} y_{i}=-\Delta y_{i}+\mathbf{v}_{i} \cdot \nabla y_{i}=q \quad \text { in } \Omega \quad \text { and }\left.\quad y_{i}\right|_{\Gamma}=\theta
$$

By the maximum principle, we have that

$$
y_{i} \geq 0 \quad \text { in } \Omega \text {. }
$$

We now decompose the function $y_{i}$ into the form $y_{i}=y_{i}^{(1)}+y_{i}^{(2)}$, where

$$
L_{i} y_{i}^{(1)}=q \quad \text { in } \Omega \quad \text { and }\left.\quad y_{i}^{(1)}\right|_{\Gamma}=0
$$


and

$$
L_{i} y_{i}^{(2)}=0 \quad \text { in } \Omega \quad \text { and }\left.\quad y_{i}^{(2)}\right|_{\Gamma}=\theta
$$

Taking the scalar product in $L^{2}(\Omega)$ of $(23)$ and the function $\left(y_{i}^{(1)}\right)^{2 k+1}$ we obtain

$$
\frac{2 k+1}{(k+1)^{2}} \int_{\Omega}\left|\nabla\left(y_{i}^{(1)}\right)^{k+1}\right|^{2} d \Omega=\int_{\Omega}\left(y_{i}^{(1)}\right)^{2 k+1} q d \Omega .
$$

This equality and the Sobolev imbedding theorem imply that

$$
\left\|\left(y_{i}^{(1)}\right)^{k}\right\|_{W_{2}^{1}(\Omega)} \leq C_{3}(k)\|q\|_{L^{2}(\Omega)}^{k} \quad \forall k \geq 1,
$$

where the constant $C_{3}(k)$ is independent of $\mathbf{v}$. From (24), by the maximum principle and the Sobolev imbedding theorem, we obtain

$$
\left\|y_{i}^{(2)}\right\|_{L^{\infty}(\Omega)} \leq C_{3}\|\theta\|_{W_{2}^{1}(\Gamma)},
$$

where $C_{3}$ is independent of $\mathbf{v}$. Moreover, due to the inequality

$$
\left\|y_{i}\right\|_{W_{2}^{s+\frac{1}{2}}(\Omega)} \leq C_{4}\left(\|q\|_{L^{2}(\Omega)}+\|\theta\|_{W_{2}^{s}(\Gamma)}+\|\mathbf{v}\|_{\left[L^{5}(\Omega)\right]^{2}}\left\|y_{i}\right\|_{W_{2}^{\frac{7}{3}}(\Omega)}\right)
$$

and standard a priori estimates for the Laplace operator, we have

$$
\left\|y_{i}\right\|_{W_{2}^{\frac{1}{2}+s}(\Omega)} \leq C_{5}\left(\left\|\mathbf{v}_{i}\right\|_{\left[L^{5}(\Omega)\right]^{2}}\right)\left(\|q\|_{L^{2}(\Omega)}+\|\theta\|_{W_{2}^{s}(\Gamma)}\right) .
$$

Hence, without the loss of generality, taking if necessary a subsequence, we can assume that

$$
y_{i} \rightarrow y \quad \text { weakly in } W_{2}^{\frac{1}{2}+s}(\Omega), \quad s \in\left(1, \frac{3}{2}\right] .
$$

In particular (22) and (28) imply that

$$
y \geq 0 \text { in } \Omega .
$$

Furthermore (28) and the inequalities (25-27) imply that

$$
\|y\|_{L^{k}(\Omega)} \leq C_{6}(k)\left(\|q\|_{L^{2}(\Omega)}+\|\theta\|_{W_{2}^{1}(\Gamma)}\right) \quad \forall k \in \mathbb{N}_{+},
$$

where the constant $C_{6}$ is independent of $\mathbf{v}$, and

$$
\|y\|_{W_{2}^{\frac{1}{2}+s}(\Omega)} \leq C_{7}\left(\|\mathbf{v}\|_{\left[L^{5}(\Omega)\right]^{2}}\right)\left(\|q\|_{L^{2}(\Omega)}+\|\theta\|_{W_{2}^{1}(\Gamma)}\right) .
$$

By (20) and (23), we can pass to the limit in (21) to obtain

$$
L y=-\Delta y+\mathbf{v} \cdot \nabla y=q \text { in } \Omega \quad \text { and }\left.\quad y\right|_{\Gamma}=\theta .
$$

Thus, we established the existence of a solution to problem (11). 
Now let us prove the uniqueness of this solution in $L^{2}(\Omega)$. Suppose that problem (11) has two solutions $y_{j} \in L^{2}(\Omega), j=1,2$. Obviously, the function $w=y_{1}-y_{2}$ is the solution to the boundary value problem

$$
L w=0 \quad \text { in } \Omega \quad \text { and }\left.\quad w\right|_{\Gamma}=0
$$

Suppose that there exists a nonzero solution to (32). Then,

$$
\left(w, L^{*} z\right)_{L^{2}(\Omega)}=0 \quad \forall z \in W_{2}^{2}(\Omega) \cap \stackrel{\circ}{W}{ }_{2}^{1}(\Omega)
$$

On the other hand, as we showed above, there exists a solution $\widetilde{z} \in W_{2}^{2}(\Omega)$ to the problem

$$
L^{*} \widetilde{z}=w \quad \text { in } \Omega \quad \text { and }\left.\quad \widetilde{z}\right|_{\Gamma}=0
$$

to obtain this solution, one merely has to exchange $\mathbf{v}$ for $\mathbf{- v}$ in (21). If we substitute $\widetilde{z}$ in $(33)$, we obtain $w \equiv 0$.

Hence, we have that $\phi(\mathbf{v})=y$ and by (29-31), we have obtained the estimates $(12-14)$.

We next consider the solution $(\mathbf{u}, p) \in\left[W_{2}^{1}(\Omega)\right]^{2} \times \widetilde{L}^{2}(\Omega)$ of the boundary value problem

$$
\begin{gathered}
-\Delta \mathbf{u}+\mathbf{v} \cdot \nabla \mathbf{u}+\boldsymbol{\omega}(\mathbf{v})+\nabla p=\mathbf{f} \quad \text { in } \Omega \\
\nabla \cdot\left(\frac{\mathbf{u}}{1+\phi(\mathbf{v})}\right)=0 \quad \text { in } \Omega,
\end{gathered}
$$

and

$$
\left.\mathbf{u}\right|_{\Gamma}=\mathbf{0}
$$

where $\phi(\mathbf{v})$ is the solution of (11) and

$$
\boldsymbol{\omega}(\mathbf{v})=\beta\left(\begin{array}{l}
0 \\
1
\end{array}\right) \frac{\phi(\mathbf{v})}{1+\phi(\mathbf{v})}
$$

By (12) and (13),

$$
\|\boldsymbol{\omega}(\mathbf{v})\|_{\left[L^{2}(\Omega)\right]^{2}} \leq C_{10}\left(\|\theta\|_{W_{2}^{1}(\Gamma)}+\|q\|_{L^{2}(\Omega)}\right)
$$

For the boundary value problem (34-36), we have the following result.

Proposition 2.4. Let $\mathbf{v} \in \mathbf{H} \cap\left[L^{5}(\Omega)\right]^{2}$ and $\mathbf{f} \in \mathbf{H}$. Then, (34-36) has a unique solution $(\mathbf{u}, p) \in\left[W_{2}^{1}(\Omega)\right]^{2} \times$ $\widetilde{L}^{2}(\Omega)$. Moreover, the estimate

$$
\left\|\frac{\mathbf{u}}{1+\phi(\mathbf{v})}\right\|_{\left[W_{2}^{1}(\Omega)\right]^{2}} \leq C_{8}\left(\|\mathbf{f}\|_{\mathbf{H}}+\|\theta\|_{W_{2}^{1}(\Gamma)}+\|q\|_{L^{2}(\Omega)}\right),
$$

where the constant $C_{8}$ is independent of $\mathbf{v}$, holds. 
Proof. We first show that if a solution $(\mathbf{u}, p) \in\left[W_{2}^{1}(\Omega)\right]^{2} \times \widetilde{L}^{2}(\Omega)$ exists, it is unique. To see this, suppose that (34-36) has two solutions $\left(\mathbf{u}_{1}, p_{1}\right),\left(\mathbf{u}_{2}, p_{2}\right) \in\left[W_{2}^{1}(\Omega)\right]^{2} \times \widetilde{L}^{2}(\Omega)$. Then, the pair $\mathbf{w}=\mathbf{u}_{1}-\mathbf{u}_{2}$ and $r=p_{1}-p_{2}$ satisfies the system of equations

$$
\begin{gathered}
-\Delta \mathbf{w}+\mathbf{v} \cdot \nabla \mathbf{w}+\nabla r=\mathbf{0} \quad \text { in } \Omega, \\
\nabla \cdot\left(\frac{\mathbf{w}}{1+\phi(\mathbf{v})}\right)=0 \quad \text { in } \Omega,
\end{gathered}
$$

and

$$
\left.\mathbf{w}\right|_{\Gamma}=\mathbf{0}
$$

Multiplying (39) by $\widetilde{\mathbf{w}}=\mathbf{w} /(1+\phi(\mathbf{v}))$, integrating over $\Omega$, and then integrating by parts, we obtain

$$
\begin{aligned}
0 & =\int_{\Omega}\left\{\nabla(1+\phi(\mathbf{v})) \widetilde{\mathbf{w}} \cdot \nabla \widetilde{\mathbf{w}}+\frac{1}{2}|\widetilde{\mathbf{w}}|^{2} \mathbf{v} \cdot \nabla(1+\phi(\mathbf{v}))\right\} d \Omega \\
& =\int_{\Omega}\left\{(1+\phi(\mathbf{v}))|\nabla \widetilde{\mathbf{w}}|^{2}+\frac{1}{2} \nabla(1+\phi(\mathbf{v})) \cdot \nabla|\widetilde{\mathbf{w}}|^{2}+\frac{1}{2}|\widetilde{\mathbf{w}}|^{2} \mathbf{v} \cdot \nabla \phi(\mathbf{v})\right\} d \Omega \\
& =\int_{\Omega}\left((1+\phi(\mathbf{v}))|\nabla \widetilde{\mathbf{w}}|^{2}-\frac{1}{2}|\widetilde{\mathbf{w}}|^{2} \Delta \phi(\mathbf{v})+\frac{1}{2}|\widetilde{\mathbf{w}}|^{2} \mathbf{v} \cdot \nabla \phi(\mathbf{v})\right) d \Omega
\end{aligned}
$$

Then, by (11),

$$
\int_{\Omega}\left((1+\phi(\mathbf{v}))|\nabla \widetilde{\mathbf{w}}|^{2}+q|\widetilde{\mathbf{w}}|^{2}\right) d \Omega=0
$$

and our statement, i.e., $\mathbf{w}=\mathbf{0}$, follows from (7) and (12).

The next step is to prove that the problem (34-36) has a solution for all $\mathbf{f} \in \mathbf{H}$. We define the linear operator $A$ by

$$
A(\mathbf{u}, p)=\left(-\Delta \mathbf{u}+\mathbf{v} \cdot \nabla \mathbf{u}+\nabla p, \frac{\nabla \cdot \mathbf{u}}{1+\phi(\mathbf{v})}-\frac{\mathbf{u} \cdot \nabla \phi(\mathbf{v})}{(1+\phi(\mathbf{v}))^{2}}\right)
$$

Obviously, for any nonnegative function $\phi(\mathbf{v}) \in W_{2}^{\frac{3}{2}}(\Omega)$, we have

$$
A \in \mathcal{L}\left(\left[W_{2}^{2}(\Omega)\right]^{2} \times \widetilde{W}_{2}^{1}(\Omega) ;\left[L^{2}(\Omega)\right]^{2} \times \widetilde{W}_{2}^{1}(\Omega)\right) .
$$

Let us now show that

$$
\operatorname{Im}(A) \supset\left[L^{2}(\Omega)\right]^{2} \times\{0\}
$$

First, we claim

$$
\overline{\operatorname{Im} \widetilde{A}}=\mathbf{V}^{*} \times \widetilde{L}^{2}(\Omega),
$$

where $(\cdot)^{*}$ denotes the dual space,

$$
\widetilde{A}(\mathbf{u}, p)=\left(\mathbf{P}(-\Delta \mathbf{u}+\mathbf{v} \cdot \nabla \mathbf{u}+\nabla p), \frac{\nabla \cdot \mathbf{u}}{1+\phi(\mathbf{v})}-\frac{\mathbf{u} \cdot \nabla \phi(\mathbf{v})}{(1+\phi(\mathbf{v}))^{2}}\right)
$$


and the operator $\mathbf{P}$ is orthoprojector from $\left[L^{2}(\Omega)\right]^{2}$ to $\mathbf{H}$. Our proof is by contradiction. Suppose that $\operatorname{Im} \widetilde{A} \neq$ $\mathbf{V}^{*} \times \widetilde{L}^{2}(\Omega)$. Then, there exists an element of the space $\mathbf{V} \times \widetilde{L}^{2}(\Omega)$ which we denote by $(\mathbf{z}, \zeta)$ such that

$$
\langle A(\mathbf{u}, p),(\mathbf{z}, \zeta)\rangle=0 \quad \forall(\mathbf{u}, p) \in\left[W_{2}^{2}(\Omega)\right]^{2} \times \widetilde{W}_{2}^{1}(\Omega)
$$

which immediately implies that

$$
\begin{gathered}
-\Delta \mathbf{z}-\mathbf{v} \cdot \nabla \mathbf{z}=\frac{1}{1+\phi(\mathbf{v})} \nabla \zeta \quad \text { in } \Omega, \\
\nabla \cdot \mathbf{z}=0 \quad \text { in } \Omega,
\end{gathered}
$$

and

$$
\left.\mathbf{z}\right|_{\Gamma}=\mathbf{0}
$$

Then, multiplying (48) by $(1+\phi(\mathbf{v})) \mathbf{z}$, integrating over $\Omega$, and then integrating by parts, we have

$$
\begin{aligned}
0 & =\int_{\Omega}\left\{(1+\phi(\mathbf{v}))|\nabla \mathbf{z}|^{2}-\frac{1}{2} \Delta \phi(\mathbf{v})|\mathbf{z}|^{2}+\frac{1}{2}(\mathbf{v} \cdot \nabla \phi(\mathbf{v}))|\mathbf{z}|^{2}\right\} d \Omega \\
& =\int_{\Omega}\left((1+\phi(\mathbf{v}))|\nabla \mathbf{z}|^{2}+q|\mathbf{z}|^{2}\right) d \Omega
\end{aligned}
$$

and, by (7) and (12), $\mathbf{z} \equiv \mathbf{0}$. Then (47) yields $\nabla \zeta=0$. Hence, $\zeta \equiv 0$. Thus, we have reached the contradiction.

Now, we let $\mathbf{f}$ be an arbitrary element from $\mathbf{H}$. Then, by (45), there exists a sequence $\left\{\left(\mathbf{f}_{i}, r_{i}, \mathbf{u}_{i}, p_{i}\right)\right\} \subset$ $\mathbf{H} \times \widetilde{L}^{2}(\Omega) \times\left[W_{2}^{2}(\Omega)\right]^{2} \times \widetilde{W}_{2}^{1}(\Omega)$ such that

$$
\begin{gathered}
\left(\mathbf{f}_{i}, r_{i}\right) \rightarrow(\mathbf{f}, 0) \quad \text { in } \mathbf{H} \times \widetilde{W}_{2}^{1}(\Omega), \\
-\Delta \mathbf{u}_{i}+\mathbf{v} \cdot \nabla \mathbf{u}_{i}+\boldsymbol{\omega}(\mathbf{v})+\nabla p_{i}=\mathbf{f}_{i} \quad \text { in } \Omega, \\
\nabla \cdot\left(\frac{\mathbf{u}_{i}}{1+\phi(\mathbf{v})}\right)=r_{i} \quad \text { in } \Omega,
\end{gathered}
$$

and

$$
\left.\mathbf{u}_{i}\right|_{\Gamma}=\mathbf{0}
$$

Multiplying (51) by $\widetilde{\mathbf{u}}_{i}=\mathbf{u}_{i} /(1+\phi(\mathbf{v}))$, integrating over $\Omega$, and then integrating by parts, we obtain, similarly to $(42)$,

$$
\int_{\Omega}\left((1+\phi(\mathbf{v}))\left|\nabla \widetilde{\mathbf{u}}_{i}\right|^{2}+q\left|\widetilde{\mathbf{u}}_{i}\right|^{2}+\frac{\boldsymbol{\omega}(\mathbf{v}) \cdot \mathbf{u}_{i}}{(1+\phi(\mathbf{v}))}\right) d \Omega-\int_{\Omega} \mathbf{f}_{i} \cdot \widetilde{\mathbf{u}}_{i} d \Omega-\int_{\Omega} r_{i} p_{i} d \Omega=0
$$

From (51) we obtain

$$
\left\|p_{i}\right\|_{L^{2}(\Omega)} \leq C_{11}\left(\left\|\mathbf{u}_{i}\right\|_{\left[W_{2}^{1}(\Omega)\right]^{2}}+\|\boldsymbol{\omega}(\mathbf{v})\|_{\left[L^{2}(\Omega)\right]^{2}}+\left\|\mathbf{f}_{i}\right\|_{\mathbf{H}}+\|\mathbf{v}\|_{\left[L^{5}(\Omega)\right]^{2}}\left\|\mathbf{u}_{i}\right\|_{\left[W_{2}^{1}(\Omega)\right]^{2}}\right) .
$$


This equality (37), and (54) imply the estimate

$$
\left\|\mathbf{u}_{i}\right\|_{\left[L^{2}(\Omega)\right]^{2}}^{2}+\left\|\frac{\mathbf{u}_{i}}{1+\phi(\mathbf{v})}\right\|_{\left[W_{2}^{1}(\Omega)\right]^{2}}^{2} \leq C_{12}\left(\|\mathbf{f}\|_{\mathbf{H}}^{2}+\|\theta\|_{W_{2}^{1}(\Gamma)}^{2}+\|q\|_{L^{2}(\Omega)}^{2}+1\right),
$$

where the constant $C_{12}$ is independent of $\mathbf{v}$. In particular, since $\phi(\mathbf{v}) \in W_{2}^{\frac{3}{2}}(\Omega)$, the estimate (55) implies that

$$
\left\|\mathbf{u}_{i}\right\|_{\left[W_{2}^{1}(\Omega)\right]^{2}}^{2} \leq C_{13}\left(\|\mathbf{f}\|_{\mathbf{H}}^{2}+\|\theta\|_{W_{2}^{1}(\Gamma)}^{2}+\|q\|_{L^{2}(\Omega)}^{2}+1\right) .
$$

Thus, we have that, taking if it is necessary a subsequence,

$$
\left(\mathbf{u}_{i}, p_{i}\right) \rightarrow(\mathbf{u}, p) \quad \text { in }\left[W_{2}^{1}(\Omega)\right]^{2} \times \widetilde{L}^{2}(\Omega) .
$$

Passing to the limit in (51) and (53), we obtain that $(\mathbf{u}, p)$ is the solution to problem (34-36). Passing to the limit in (55), we obtain (38).

We are now in a position to prove Theorem 2.1 by a fixed point argument.

Proof of Theorem 2.1. We first prove the theorem under the assumption that $\theta \in W_{2}^{s}(\Gamma), s \in\left(1, \frac{3}{2}\right]$.

We define the mapping $G(\mathbf{v})$ by

$$
G(\mathbf{v})=\frac{\mathbf{u}}{1+\phi(\mathbf{v})}
$$

where $\phi(\mathbf{v})$ is the solution of $(11)$ and $(\mathbf{u}, p) \in\left[W_{2}^{1}(\Omega)\right]^{2} \times \widetilde{L}^{2}(\Omega)$ is the solution of the boundary value problem (34-36). Note that by (36) and (55), $G(\mathbf{v}) \in \mathbf{V}$. Obviously, if $\widetilde{\mathbf{v}}$ is the fixed point of the mapping $G$, then the pair $((1+\phi(\widetilde{\mathbf{v}})) \widetilde{\mathbf{v}}, \phi(\widetilde{\mathbf{v}}))$ is the solution to problem $(1-6)$.

Let us now prove that

$$
G \in C\left(\mathbf{H} \cap\left[L^{5}(\Omega)\right]^{2} ; \mathbf{H} \cap\left[L^{5}(\Omega)\right]^{2}\right) .
$$

To prove (58), let us establish the continuity of the mapping $\phi$, i.e.,

$$
\phi \in C\left(\mathbf{H} \cap\left[L^{5}(\Omega)\right]^{2} ; L^{2}(\Omega)\right) .
$$

Suppose the contrary. In that case, there exists a sequence $\mathbf{v}_{i}$ such that

$$
\mathbf{v}_{i} \rightarrow \mathbf{v} \quad \text { in } \mathbf{H} \cap\left[L^{5}(\Omega)\right]^{2}
$$

and

$$
\phi\left(\mathbf{v}_{i}\right) \nrightarrow \phi(\mathbf{v}) \quad \text { in } L^{2}(\Omega) .
$$

By (14) and (60), we can assume

$$
\phi\left(\mathbf{v}_{i}\right) \rightarrow \widetilde{y} \quad \text { in } W_{4}^{1}(\Omega) .
$$

Passing to the limit in (11), keeping in mind (60) and (61), we have that

$$
L \widetilde{y}=q \quad \text { in } \Omega \quad \text { and }\left.\quad \widetilde{y}\right|_{\Gamma}=\theta .
$$


Then, the uniqueness of the solution to problem $(11)$ in $L^{2}(\Omega)$ implies immediately that $\widetilde{y}=\phi(\mathbf{v})$. We have reached the contradiction.

By (59), to prove (58) it now suffices to show that the mapping $G_{1}(\mathbf{v})=\mathbf{u}$, where $\mathbf{u}$ is the solution of $(34-36)$, belongs to $C\left(\mathbf{H} \cap\left[L^{5}(\Omega)\right]^{2} ;\left[L^{5}(\Omega)\right]^{2}\right)$. Suppose the contrary. In that case, there exists the sequence $\mathbf{v}_{i}$ such that

$$
\mathbf{v}_{i} \rightarrow \mathbf{v} \quad \text { in } \mathbf{H} \cap\left[L^{5}(\Omega)\right]^{2}
$$

and

$$
G_{1}\left(\mathbf{v}_{i}\right) \nrightarrow G_{1}(\mathbf{v}) \quad \text { in }\left[L^{2}(\Omega)\right]^{2} .
$$

By $(55,59)$, and $(62)$, we can assume that

$$
G_{1}\left(\mathbf{v}_{i}\right) \rightarrow \widetilde{\mathbf{u}} \quad \text { in }\left[W_{2}^{1}(\Omega)\right]^{2}
$$

and

$$
\boldsymbol{\omega}\left(\mathbf{v}_{i}\right) \rightarrow \boldsymbol{\omega}(\mathbf{v}) \quad \text { in }\left[L^{2}(\Omega)\right]^{2}
$$

Thus, passing to the limit in (57-34), we obtain that

$$
\begin{gathered}
-\Delta \widetilde{\mathbf{u}}+\mathbf{v} \cdot \nabla \widetilde{\mathbf{u}}+\boldsymbol{\omega}(\mathbf{v})+\nabla \widetilde{p}=\mathbf{f} \quad \text { in } \Omega, \\
\nabla \cdot\left(\frac{\widetilde{\mathbf{u}}}{1+\phi(\mathbf{v})}\right)=0 \quad \text { in } \Omega,
\end{gathered}
$$

and

$$
\left.\widetilde{\mathbf{u}}\right|_{\Gamma}=\mathbf{0}
$$

On the other hand, for the element $\mathbf{u}=G_{1}(\mathbf{v})$ one can find the pressure $p \in L^{2}(\Omega)$ such that the pair (u, $p$ ) satisfies (34-36). Then, Proposition 2.4 implies that $\widetilde{\mathbf{u}}=G_{1}(\mathbf{v})$. Thus, we have arrived at a contradiction.

Let $B_{\gamma}$ be the ball in $\mathbf{H} \cap\left[L^{5}(\Omega)\right]^{2}$ of radius $\gamma$ centered at zero. By (38) and the Sobolev embedding theorem, for all $\gamma$ large enough,

$$
G\left(B_{\gamma}\right) \subset B_{\gamma}
$$

Moreover, (45) and the compactness of the embedding of $\mathbf{V} \subset \mathbf{H} \cap\left[L^{5}(\Omega)\right]^{2}$ implies that

$$
\operatorname{Im} G \quad \text { is compact in } \quad \mathbf{H} \cap\left[L^{5}(\Omega)\right]^{2} .
$$

Thus, all conditions of the Leray-Shauder fixed point theorem hold true and there exists a fixed point of the mapping $G$. By (14), the temperature component of our solution $T \in W_{2}^{\frac{1}{2}+s}(\Omega)$. 
Now let us prove that the component $\mathbf{u}$ of our solution belongs to $\left[W_{2}^{s+\frac{1}{2}}(\Omega)\right]^{2}$. First, we rewrite the system (1-3) in the form

$$
\begin{gathered}
-\Delta \mathbf{u}+\nabla p=\widetilde{\mathbf{f}} \quad \text { in } \Omega, \\
\nabla \cdot \mathbf{u}=\widetilde{m} \quad \text { in } \Omega, \\
\left.\mathbf{u}\right|_{\Gamma}=\mathbf{0},
\end{gathered}
$$

where

$$
\widetilde{\mathbf{f}}=-\rho \mathbf{u} \cdot \nabla \mathbf{u}-\beta\left(\begin{array}{l}
0 \\
1
\end{array}\right) \rho T
$$

and $\widetilde{m}=-(\mathbf{u} \cdot \nabla \rho) / \rho$. By $(14)$ and $(38)$,

$$
\|\widetilde{\mathbf{f}}\|_{\left[W_{4}^{-1}(\Omega)\right]^{2}}+\|\widetilde{m}\|_{L^{4-\varepsilon}(\Omega)} \leq C_{14}(\varepsilon)\left(\|\theta\|_{W_{2}^{1}(\Gamma)}^{2}+\|q\|_{L^{2}(\Omega)}^{2}+\|\mathbf{f}\|_{\mathbf{H}}^{2}+1\right) \quad \forall \varepsilon>0 .
$$

Then, by the theorem on regularity of the Stokes system (see [8], Prop. 2.3.3, p. 35) we have

$$
\|\mathbf{u}\|_{\left[W_{4-\varepsilon}^{1}(\Omega)\right]^{2}}+\|p\|_{L^{4-\varepsilon}(\Omega)} \leq C_{14}(\varepsilon)\left(\|\theta\|_{W_{2}^{1}(\Gamma)}^{2}+\|q\|_{L^{2}(\Omega)}^{2}+\|\mathbf{f}\|_{\mathbf{H}}^{2}+1\right) \quad \forall \varepsilon>0 .
$$

This improved regularity implies immediately that

$$
\|\widetilde{\mathbf{f}}\|_{\left[L^{2}(\Omega)\right]^{2}}+\|\widetilde{m}\|_{W_{2}^{s-\frac{1}{2}}(\Omega)} \leq C_{15}(\varepsilon)\left(\|\theta\|_{W_{2}^{s}(\Gamma)}^{4}+\|q\|_{L^{2}(\Omega)}^{4}+\|\mathbf{f}\|_{\mathbf{H}}^{4}+1\right) .
$$

Hence by regularity results for the Stokes system (see, e.g. [8], Prop. 2.2, p. 33), we have

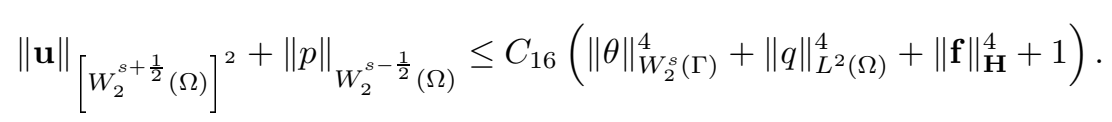

To establish the $\left[W_{2}^{2}(\Omega)\right]^{2}$ regularity of the function $\mathbf{w}=\rho \mathbf{u}$, we rewrite $(1-3)$ in terms of $(\mathbf{w}, \zeta)$ that satisfies the equations

$$
\begin{gathered}
-\Delta \mathbf{w}+\nabla \zeta=\mathbf{g} \quad \text { in } \Omega, \\
\nabla \cdot \mathbf{w}=0 \quad \text { in } \Omega,
\end{gathered}
$$

and

$$
\left.\mathbf{w}\right|_{\Gamma}=\mathbf{0},
$$

where

$$
\mathbf{g}=\rho\left(-\rho \mathbf{u} \cdot \nabla \mathbf{u}-\beta\left(\begin{array}{c}
0 \\
1
\end{array}\right) \rho T+\mathbf{f}+2 \nabla T \cdot \nabla \mathbf{w}-(q-\rho \mathbf{u} \cdot \nabla T) \mathbf{w}\right)+p \nabla \rho
$$


Since by $(14,70) \mathbf{g} \in\left[L^{2}(\Omega)\right]^{2}$, Proposition 2.2 yields $\mathbf{w} \in \mathbf{H}^{2}$.

Now, let us prove the estimate (10). Let $\mathbf{u}$ be an arbitrary solution to problem (1-6) and the parameter $s \in\left[1, \frac{3}{2}\right]$. Since $\rho \mathbf{u} \in \mathbf{H} \cap\left[L^{5}(\Omega)\right]^{2}$ and $\nabla \cdot(\rho \mathbf{u})=0$, we can use the a priori estimate (13) for the boundary problem (11), i.e.,

$$
\|T\|_{L^{2}(\Omega)} \leq C_{17}\left(\|q\|_{L^{2}(\Omega)}+\|\theta\|_{W_{2}^{1}(\Gamma)}\right) .
$$

Thus, for the function $\mathbf{w}_{1}=-\beta\left(\begin{array}{c}0 \\ \rho T\end{array}\right)$, the analog of (37) holds true, i.e.,

$$
\left\|\mathbf{w}_{1}\right\|_{\left[L^{2}(\Omega)\right]^{2}} \leq C_{18}\left(\|\theta\|_{W_{2}^{1}(\Gamma)}+\|q\|_{L^{2}(\Omega)}+1\right) .
$$

Taking the scalar product in $L^{2}(\Omega)$ of (1) and the function $\rho \mathbf{u}$, similar to (54), we have

$$
\int_{\Omega}\left(\frac{1}{\rho}|\nabla(\rho \mathbf{u})|^{2}+\rho \mathbf{u} \cdot \mathbf{w}_{1}\right) d \Omega-\int_{\Omega} \rho \mathbf{u} \cdot \mathbf{f} d \Omega=0 .
$$

From (1.64) and this equality we have

$$
\|\mathbf{u}\|_{\left[W_{2}^{1}(\Omega)\right]^{2}}^{2} \leq C_{19}\left(\|\mathbf{f}\|_{\mathbf{H}}^{2}+\|\theta\|_{W_{2}^{1}(\Gamma)}^{2}+\|q\|_{L^{2}(\Omega)}^{2}+1\right) .
$$

We decompose the function $T$ in the form $T=\tau_{1}+\tau_{2}$, where

$$
-\Delta \tau_{2}=q \quad \text { in } \Omega \quad \text { and }\left.\quad \tau_{2}\right|_{\Gamma}=\theta .
$$

By (63) and standard a priori estimates for the Laplace operator (see, e.g. [5]),

$$
\left\|\tau_{2}\right\|_{W_{2}^{s+1 / 2}(\Omega)} \leq C_{20}\left(\|\theta\|_{W_{2}^{s}(\Gamma)}+\|q\|_{L^{2}(\Omega)}\right) .
$$

By (4) and (64), the function $\tau_{1}$ satisfies

$$
-\Delta \tau_{1}+\rho \mathbf{u} \cdot \nabla \tau_{1}=-\rho \mathbf{u} \cdot \nabla \tau_{2} \quad \text { in } \Omega \quad \text { and }\left.\quad \tau_{1}\right|_{\Gamma}=0 .
$$

Due to the inequality $\left\|\rho \mathbf{u} \cdot \nabla \tau_{2}\right\|_{L^{2}(\Omega)} \leq C_{21}\|\mathbf{u}\|_{\left[W_{2}^{1}(\Omega)\right]^{2}}\left\|\tau_{2}\right\|_{W_{2}^{\frac{5}{4}}(\Omega)}$ and standard a priori estimates for elliptic equations, we have

$$
\left\|\tau_{1}\right\|_{W_{2}^{2}(\Omega)} \leq C_{22}\|\mathbf{u}\|_{\left[W_{2}^{1}(\Omega)\right]^{2}}\left\|\tau_{2}\right\|_{W_{2}^{\frac{5}{4}}(\Omega)} .
$$

The inequalities (76-80) yield

$$
\|T\|_{W_{2}^{\frac{1}{2}+s}(\Omega)} \leq C_{23}\left(\|\mathbf{f}\|_{\mathbf{H}}^{2}+\|\theta\|_{W_{2}^{1}(\Gamma)}^{2}+\|q\|_{L^{2}(\Omega)}^{2}+1\right) .
$$

Finally, repeating the argument extending over (64-70), we obtain from (10) and (81) the $W_{2}^{s+\frac{1}{2}}(\Omega)$-norm estimate for the function $\mathbf{u}$.

Now let $\theta$ be an arbitrary nonnegative function from the space $W_{2}^{1}(\Gamma)$. Due to the continuous embedding of the space $W_{2}^{1}(\Gamma)$ in $C^{0}(\Gamma)$, we can approximate this function by the nonnegative functions $\theta_{i} \in W_{2}^{2}(\Gamma)$, i.e.,

$$
\theta_{i} \rightarrow \theta \quad \text { in } W_{2}^{1}(\Gamma) .
$$


For each element $\left(\mathbf{f}, q, \theta_{i}\right)$, there exists at least one solution $\left(\mathbf{u}_{i}, T_{i}, p_{i}\right) \in\left[W_{2}^{2}(\Omega)\right]^{2} \times W_{2}^{2}(\Omega) \times \widetilde{W}_{2}^{1}(\Omega)$ to the problem (1-6). Moreover, by (10), the sequence $\left\{\left(\mathbf{u}_{i}, T_{i}, p_{i}\right)\right\}_{i=1}^{\infty}$ is bounded in $\left[W_{2}^{\frac{3}{2}}(\Omega)\right]^{2} \times W_{2}^{\frac{3}{2}}(\Omega) \times \widetilde{W}_{2}^{\frac{1}{2}}(\Omega)$. Taking the subsequence which converges weakly in $\left[W_{2}^{\frac{3}{2}}(\Omega)\right]^{2} \times W_{2}^{\frac{3}{2}}(\Omega) \times \widetilde{W}_{2}^{\frac{1}{2}}(\Omega)$ to some element $(\mathbf{u}, T, p)$ and passing to the limit in (1-6), we can show that this element is solution to (1-6). The proof of Theorem 2.1 is complete.

\section{The optimal CONTROL PROBLEM}

In this section, we consider the optimal control problem

$$
\begin{gathered}
J(\mathbf{u}, \theta)=\frac{1}{2}\left\|\mathbf{u}-\mathbf{u}_{0}\right\|_{\left[L^{2}(\Omega)\right]^{2}}^{2}+\frac{1}{2}\left\|\theta-\theta_{0}\right\|_{W_{2}^{1}(\Gamma)}^{2} \longrightarrow \inf \\
(\mathbf{u}, p, T, \theta) \text { satisfies }(1-7)
\end{gathered}
$$

and

$$
\theta \in K
$$

where $K \subset\left\{\theta \in W_{2}^{1}(\Omega) \mid \theta(\mathbf{x}) \geq 0 \forall \mathbf{x} \in \Gamma\right\}$ is a convex, closed, nonempty set in $W_{2}^{1}(\Gamma)$ and

$$
\|\theta\|_{W_{2}^{1}(\Gamma)}^{2}=\|\theta\|_{L^{2}(\Gamma)}^{2}+\left\|\frac{\partial \theta}{\partial \vec{\tau}}\right\|_{L^{2}(\Gamma)}^{2}
$$

with $\vec{\tau}$ denoting the unit, continuous tangent vector to $\Gamma$. The functions $\mathbf{u}_{0} \in\left[L^{2}(\Omega)\right]^{2}$ and $\theta_{0} \in W_{2}^{1}(\Gamma)$ are given; the function $\theta$ is the control to be determined as part of the solution of the optimal control problem.

We say that $(\mathbf{u}, T, p, \theta) \in\left[W_{2}^{2}(\Omega)\right]^{2} \times W_{2}^{\frac{3}{2}}(\Omega) \times \widetilde{L}^{2}(\Omega) \times L^{2}(\Gamma)$ is an admissible element to the extremal problem (82-84) if (83-84) hold true. We denote the all set of admissible elements to the problem (82-84) by $\mathcal{U}_{a d}$.

We say that an admissible element $(\widehat{\mathbf{u}}, \widehat{T}, \widehat{p}, \widehat{q}) \in\left[\left(W_{2}^{2}(\Omega)\right]^{2} \times W_{2}^{\frac{3}{2}}(\Omega) \times \widetilde{L}^{2}(\Omega) \times L^{2}(\Gamma)\right.$ is a global minimizer of the problem (82-84) if

$$
J(\widehat{u}, \widehat{g}) \leq J(u, g) \quad \forall(\mathbf{u}, T, p, g) \in \mathcal{U}_{a d}
$$

We have the following result.

Theorem 3.1. Let $\mathbf{f} \in\left[L^{2}(\Omega)\right]^{2}, q \in L^{2}(\Omega), \mathbf{u}_{0} \in\left[L^{2}(\Omega)\right]^{2}$, and $\theta_{0} \in W_{2}^{1}(\Gamma)$ be given and let condition (7) hold true. Then, there exists a solution $(\widehat{\mathbf{u}}, \widehat{T}, \widehat{p}, \widehat{g})$ of the problem $(82-84)$ belonging to $\left[W_{2}^{\frac{3}{2}}(\Omega)\right]^{2} \times W_{2}^{\frac{3}{2}}(\Omega) \times$ $\widetilde{L}^{2}(\Omega) \times L^{2}(\Gamma)$ such that $\widehat{\mathbf{u}} /(1+\widehat{T}) \in \mathbf{H}^{2}$.

Proof. By Theorem 2.1, there exists at least one admissible element $(\mathbf{u}, T, p, \theta) \in\left[W_{2}^{\frac{3}{2}}(\Omega)\right]^{2} \times W_{2}^{\frac{3}{2}}(\Omega) \times \widetilde{L}^{2}(\Omega) \times$ $W_{2}^{1}(\Gamma)$ to problem (82-84) with $\rho \mathbf{u} \in \mathbf{H}^{2}$. Thus, there exists a minimizing sequence $\left\{\left(\mathbf{u}_{i}, T_{i}, p_{i}, \theta_{i}\right)\right\}_{i=1}^{\infty} \in$ $\underset{\text { sequence }}{\left[W_{2}^{\frac{3}{2}}(\Omega)\right]^{2}} \times W_{2}^{\frac{3}{2}}(\Omega) \times \widetilde{L}^{2}(\Omega) \times W_{2}^{1}(\Gamma)$ such that $\rho_{i} \mathbf{u}_{i} \in \mathbf{H}^{2}$. Let $\mathbf{v}_{i}=\rho_{i} \mathbf{u}_{i} \in \mathbf{H}^{2}$. Then, by Theorem 2.1, the

$$
\left\{\left(\mathbf{v}_{i}, \mathbf{u}_{i}, T_{i}, p_{i}, \theta_{i}\right)\right\}_{i=1}^{\infty} \quad \text { is bounded in } \mathbf{H}^{2} \times\left[W_{2}^{\frac{3}{2}}(\Omega)\right]^{2} \times W_{2}^{\frac{3}{2}}(\Omega) \times \widetilde{L}^{2}(\Omega) \times L^{2}(\Gamma)
$$


Thus, taking if necessary a subsequence, we have that

$$
\left(\mathbf{v}_{i}, \mathbf{u}_{i}, T_{i}, p_{i}, \theta_{i}\right) \rightarrow(\widehat{\mathbf{v}}, \widehat{\mathbf{u}}, \widehat{T}, \widehat{p}, \widehat{\theta}) \quad \text { weakly in } \quad \mathbf{H}^{2} \times\left[W_{2}^{\frac{3}{2}}(\Omega)\right]^{2} \times W_{2}^{\frac{3}{2}}(\Omega) \times \widetilde{L}^{2}(\Omega) \times L^{2}(\Gamma)
$$

Obviously, $(\widehat{\mathbf{u}}, \widehat{T}, \widehat{p}, \widehat{\theta})$ satisfies the system (1-4). Moreover, since the set $K$ is closed in the weak topology in $W_{2}^{1}(\Gamma)$, the function $\widehat{\theta}$ belongs to the set $K$. Hence, $(\widehat{\mathbf{u}}, \widehat{p}, \widehat{\theta})$ is the admissible element to problem (82-84). Then, the lower semicontinuity of the functional $J$ respect to weak convergence in $\left[L^{2}(\Omega)\right]^{2} \times L^{2}(\Gamma)$ yields that $(\widehat{\mathbf{u}}, \widehat{T}, \widehat{p}, \widehat{\theta})$ is the solution to problem $(82-84)$.

Now we would like to study the question of the uniqueness of the solution to problem (82-84). First, let us reformulate the extremal problem $(82-84)$ as a problem of finding the point of least distance between $\left(\mathbf{u}_{0}, \theta_{0}\right)$ and some set in the space $\left[L^{2}(\Omega)\right]^{2} \times W_{2}^{1}(\Gamma)$. Let us introduce the set

$$
\begin{aligned}
\mathcal{T}=\{(\mathbf{u}, \theta) \in & {\left[W_{2}^{1}(\Omega)\right]^{2} \times W_{2}^{1}(\Gamma) \mid \theta \in K, \text { and there exist a pair }(T, p) \in W_{2}^{1}(\Omega) \times \widetilde{L}^{2}(\Omega) } \\
& \text { such that } \left.(\mathbf{u}, p, T) \text { satisfies }(1)-(4),\left.\quad T\right|_{\Gamma}=\theta, \text { and } \rho(1+T)=1\right\} .
\end{aligned}
$$

Obviously, if the element $(\widehat{\mathbf{u}}, \widehat{p}, \widehat{T}, \widehat{\theta})$ is a solution to problem $(82-84)$, then the pair $(\widehat{\mathbf{u}}, \widehat{\theta})$ is solution to the following problem:

$$
\left\|(\mathbf{u}, \theta)-\left(\mathbf{u}_{0}, \theta_{0}\right)\right\|_{\left[L^{2}(\Omega)\right]^{2} \times W_{2}^{1}(\Gamma)} \rightarrow \inf , \quad(\mathbf{u}, \theta) \in \mathcal{T}
$$

Let us recall some facts about problems of the type (87). First, let $X$ be a Banach space and $M \subset X$. We say that the set $M$ is sequentially weakly closed if every point in the space $X$ that is the weak limit of some sequence of elements $\left\{y_{n}\right\}_{1}^{\infty} \subset M$ belongs to $M$. Second, let $X$ be a Banach space and $M \subset X$. We call a set $M$ a Tchebycheff set if for any $f \in X$ there exists a unique element $\widehat{x} \in M$ satisfying the condition $\|f-\widehat{x}\|=\inf _{x \in M}\|f-x\|$.

We will need the following particular case of Efimov-Stechkin theorem (see, e.g. [2]).

Theorem 3.2. Let $X$ be a Hilbert space and let $M \subset X$ be a Tchebycheff set. Then, if $M$ is sequentially weakly closed, it is convex.

We then have the following result.

Theorem 3.3. Let $f \in\left[\left(L^{2}(\Omega)\right]^{2}\right.$ and $q \in L^{2}(\Omega)$. Then, there exists a set $\mathcal{O} \subset\left[L^{2}(\Omega)\right]^{2} \times W_{2}^{1}(\Gamma)$ such that for all $\left(\mathbf{u}_{0}, \theta_{0}\right) \in \mathcal{O}$, the problem $(82-84)$ has a unique solution. On the other hand, if the set $\mathcal{T}$ is not convex, there exists a pair $\left(\mathbf{u}_{0}, \theta_{0}\right)$ such that the problem (82-84) has more than one solution.

Proof. Let $\left\{\left(\mathbf{u}_{i}, \theta_{i}\right)\right\}_{i=1}^{\infty} \subset \mathcal{T}$ be an arbitrary sequence in $\left[L^{2}(\Omega)\right]^{2} \times W_{2}^{1}(\Gamma)$ such that

$$
\left(\mathbf{u}_{i}, \theta_{i}\right) \rightarrow(\mathbf{u}, \theta) \quad \text { weakly in }\left[L^{2}(\Omega)\right]^{2} \times W_{2}^{1}(\Gamma)
$$

By the definition of the set $\mathcal{T}$, there exists a sequence $\left\{T_{i}, p_{i}\right\} \in W_{2}^{1}(\Omega) \times \widetilde{L}^{2}(\Omega)$ such that the element $\left(\mathbf{u}_{i}, T_{i}, p_{i}, \theta_{i}\right)$ satisfies (1-4) and $\left.T_{i}\right|_{\Gamma}=\theta_{i}$. On the other hand, by the a priori estimate (10), the sequence $\left\{\left(\mathbf{u}_{i}, T_{i}, p_{i}, \theta_{i}\right)\right\}$ is bounded in $\left[W_{2}^{\frac{3}{2}}(\Omega)\right]^{2} \times W_{2}^{\frac{3}{2}}(\Omega) \times \widetilde{L}^{2}(\Omega) \times W_{2}^{1}(\Gamma)$ and, taking if necessary a subsequence, we have that

$$
\left(\mathbf{u}_{i}, T_{i}, p_{i}, \theta_{i}\right) \rightarrow(u, T, p, \theta) \quad \text { weakly in }\left[W_{2}^{\frac{3}{2}}(\Omega)\right]^{2} \times W_{2}^{\frac{3}{2}}(\Omega) \times \widetilde{L}^{2}(\Omega) \times W_{2}^{1}(\Gamma)
$$

Exacty in the same way as was done in the proof of Theorem 3.1, one can show that $(\mathbf{u}, T, p, \theta)$ is an admissible element to the extremal problem $(82-84)$. Thus, $(\mathbf{u}, \theta) \in \mathcal{T}$ and the set $\mathcal{T}$ is sequentially weakly closed. Then, 
if the set $\mathcal{T}$ is not convex, by Theorem 3.2 there exists the pair $\left(\mathbf{u}_{0}, \theta_{0}\right) \in\left[L^{2}(\Omega)\right]^{2} \times W_{2}^{1}(\Gamma)$ such that the problem (87) has at least two optimal solutions $\left(\widehat{\mathbf{u}}_{j}, \widehat{\theta}_{j}\right), j=1,2$. Then, by the definition of the set $\mathcal{T}$, there exist $\left(\widehat{T}_{j}, \widehat{p}_{j}\right), j=1,2$, such that the elements $\left(\widehat{\mathbf{u}}_{j}, \widehat{T}_{j}, \widehat{p}_{j}, \widehat{\theta}_{j}\right)$ are admissible elements to problem (82-84). Obviously, these elements are also solutions to $(82-84)$.

In [9], it is proved that a dense set $\mathcal{O}$ in $\left[L^{2}(\Omega)\right]^{2} \times W_{2}^{1}(\Gamma)$ exists such that problem (87) has a unique solution. Let $\left(\mathbf{u}_{0}, \theta_{0}\right) \in \mathcal{O}$ be an arbitrary point. Suppose that the problem (82-84) has two solutions $\left(\widehat{\mathbf{u}}_{j}, \widehat{T}_{j}, \widehat{p}_{j}, \widehat{\theta}_{j}\right)$, $j=1,2$. In that case, $\widehat{\mathbf{u}}_{1}=\widehat{\mathbf{u}}_{2}$ and $\widehat{\theta}_{1}=\widehat{\theta}_{2}$. Also, we can rewrite the equation $\nabla \cdot\left(\mathbf{u}_{j} \rho_{j}\right)=0$ in the form $\nabla \cdot \mathbf{u}_{j}=\rho_{j} \mathbf{u}_{j} \cdot \nabla T_{j}$. Thus, by (3), $\Delta T_{1}=\Delta T_{2}$ and the uniqueness of the solution to Dirichlet problem for the Laplace operator implies that $T_{1}=T_{2}$. Then (1) yields $p_{1}=p_{2}$.

\section{The Optimality SYSTEM}

To write out the optimality system for the problem (82-84), it is convienient for us to rewrite this problem in a slightly different form. Instead of the variable $\mathbf{u}$, we introduce the new variable $\mathbf{v}=\rho \mathbf{u}$. Then, (82-84) are transformed into

$$
\begin{gathered}
\mathbf{F}_{1}(\mathbf{v}, T, p)=-(1+T) \Delta \mathbf{v}-2 \nabla T \cdot \nabla \mathbf{v}+(1+T) \mathbf{v} \cdot \nabla \mathbf{v}+\beta\left(\begin{array}{c}
0 \\
1
\end{array}\right) \rho T+\nabla p+q \mathbf{v}-\mathbf{f}=\mathbf{0} \quad \text { in } \Omega, \\
\nabla \cdot \mathbf{v}=0 \quad \text { in } \Omega, \\
\rho(1+T)=1 \quad \text { in } \Omega, \\
\left.\mathbf{v}\right|_{\Gamma}=\mathbf{0}, \\
F_{2}(\mathbf{v}, T, p)=-\Delta T+\mathbf{v} \cdot \nabla T-q=0 \quad \text { in } \Omega, \\
\left.T\right|_{\Gamma}=\theta,
\end{gathered}
$$

and

$$
\theta \in K
$$

In terms of the new variables, the functional $J$ has the form

$$
\widetilde{J}(\mathbf{v}, T, \theta)=\frac{1}{2}\left\|(1+T) \mathbf{v}-\mathbf{u}_{0}\right\|_{\left[L^{2}(\Omega)\right]^{2}}^{2}+\frac{1}{2}\left\|\theta-\theta_{0}\right\|_{W_{2}^{1}(\Gamma)}^{2} .
$$

We then have the following result: 
Theorem 4.1. Suppose that $\mathbf{f} \in\left(L^{2}(\Omega)\right)^{2}, q \in L^{2}(\Omega), \mathbf{u}_{0} \in\left[L^{2}(\Omega)\right]^{2}, \theta_{0} \in W_{2}^{1}(\Gamma)$, and condition (8) holds true. Let $(\widehat{\mathbf{u}}, \widehat{T}, \widehat{p}, \widehat{g})$ be the optimal solution to the problem (82-84). Then, there exists a Lagrange multiplier $(\lambda, \mathbf{w}, \sigma, R) \in \mathbb{R}^{1} \times \mathbf{H}^{2} \times \widetilde{W}_{2}^{1}(\Omega) \times W_{2}^{2}(\Omega)$ such that

$$
\begin{aligned}
& (\lambda, \mathbf{w}, \sigma, R) \neq 0, \\
& -\Delta((1+\widehat{T}) \mathbf{w})-\widehat{\mathbf{v}} \cdot \nabla((1+\widehat{T}) \mathbf{w})+(1+\widehat{T}) \mathbf{w} \cdot(\nabla \widehat{\mathbf{v}})^{T}+2 \nabla \widehat{T} \cdot \nabla \mathbf{w}+q \mathbf{w} \\
& +R \nabla T+2 \mathbf{w} \Delta \widehat{T}+\nabla \sigma+\left(\widehat{\mathbf{v}}-\mathbf{u}_{0}\right)(1+\widehat{T})=\mathbf{0} \quad \text { in } \Omega, \\
& \nabla \cdot \mathbf{w}=0 \quad \text { in } \Omega, \\
& \left.\mathbf{w}\right|_{\Gamma}=\mathbf{0}, \\
& -\Delta R-\widehat{\mathbf{v}} \cdot \nabla R+\frac{\beta}{(1+\widehat{T})^{2}}\left(\begin{array}{c}
0 \\
1
\end{array}\right) \cdot \mathbf{w}+\mathbf{w} \cdot \Delta \widehat{\mathbf{v}}+2 \nabla \mathbf{w}: \nabla \widehat{\mathbf{v}}+R(\widehat{\mathbf{v}} \cdot \nabla \widehat{\mathbf{v}}) \cdot \mathbf{w}+\left(\widehat{\mathbf{u}}-\mathbf{u}_{0}\right) \cdot \widehat{\mathbf{v}}=0 \quad \text { in } \Omega \\
& \widehat{\mathbf{v}}=\widehat{\mathbf{u}} /(1+\widehat{T}) \text { in } \Omega \\
& \left.R\right|_{\Gamma}=0, \\
& \left(-\lambda \Delta_{\Gamma}\left(\widehat{\theta}-\theta_{0}\right)+\lambda\left(\widehat{\theta}-\theta_{0}\right)+\frac{\partial R}{\partial \nu}, \theta-\widehat{\theta}\right)_{L^{2}(\Gamma)} \geq 0 \quad \forall \theta \in K
\end{aligned}
$$

where $\nabla \mathbf{w}: \nabla \widehat{\mathbf{v}}=\sum_{k=1}^{2} \nabla w_{k} \cdot \nabla \widehat{v}_{k}$ and $\Delta_{\Gamma}=\partial^{2} / \partial \vec{\tau}^{2}$.

Proof. We will obtain the optimality system (97-104) using the penalization method. Let us consider the auxiliary extremal problem

$$
\begin{gathered}
J_{\varepsilon}(\mathbf{v}, T, p, \theta)=\widetilde{J}(\mathbf{v}, T, \theta)+\frac{1}{2 \varepsilon}\left\|\mathbf{F}_{1}(\mathbf{v}, T, p)\right\|_{\left[L^{2}(\Omega)\right]^{2}}^{2}+\frac{1}{2 \varepsilon}\left\|F_{2}(\mathbf{v}, T, p)\right\|_{L^{2}(\Omega)}^{2} \\
+\frac{1}{2}\|\mathbf{v}-\widehat{\mathbf{v}}\|_{\left[L^{2}(\Omega)\right]^{2}}^{2}+\frac{1}{2}\|T-\widehat{T}\|_{L^{2}(\Omega)}^{2} \rightarrow \inf , \\
\nabla \cdot \mathbf{v}=0 \quad \text { in } \Omega, \\
\left.\mathbf{v}\right|_{\Gamma}=\mathbf{0}, \\
\left.T\right|_{\Gamma}=\theta,
\end{gathered}
$$

and

$$
\theta \in K
$$


where $\varepsilon>0$ is a positive parameter and $(\widehat{\mathbf{v}}(1+\widehat{T}), \widehat{T}, \widehat{p}, \widehat{\theta})$ is the solution to the extremal problem (82-84). The existence of this solution was established in Theorem 3.1. By a method similar to one used in the proof of Theorem 3.1, one can prove that there exists at least one solution $\left(\widehat{\mathbf{v}}_{\varepsilon}, \widehat{T}_{\varepsilon}, \widehat{p}_{\varepsilon}, \widehat{\theta}_{\varepsilon}\right) \in \mathbf{H}^{2} \times W_{2}^{\frac{3}{2}}(\Omega) \times \widetilde{W}_{2}^{1}(\Omega) \times W_{2}^{1}(\Gamma)$ of the problem (105-109). Moreover, since $J_{\varepsilon}\left(\widehat{\mathbf{v}}_{\varepsilon}, \widehat{T}_{\varepsilon}, \widehat{p}_{\varepsilon}, \widehat{\theta}_{\varepsilon}\right) \leq J_{\varepsilon}(\widehat{\mathbf{v}}, \widehat{T}, \widehat{p}, \widehat{\theta})=\widetilde{J}(\widehat{\mathbf{v}}, \widehat{T}, \widehat{\theta})$, by Theorem 2.1 we have

$$
\left\{\left(\widehat{\mathbf{v}}_{\varepsilon}, \widehat{T}_{\varepsilon}, \widehat{p}_{\varepsilon}, \widehat{\theta}_{\varepsilon}\right)\right\}_{\varepsilon \in(0,1)} \text { is bounded in } \mathbf{H}^{2} \times W_{2}^{\frac{3}{2}}(\Omega) \times \widetilde{W}_{2}^{1}(\Omega) \times W_{2}^{1}(\Gamma)
$$

Thus, without the loss of generality, taking if necessary a subsequence, we have that

$$
\left(\widehat{\mathbf{v}}_{\varepsilon}, \widehat{T}_{\varepsilon}, \widehat{p}_{\varepsilon}, \widehat{\theta}_{\varepsilon}\right) \rightarrow(\widetilde{\mathbf{v}}, \widetilde{T}, \widetilde{p}, \widetilde{\theta}) \quad \text { weakly in } \mathbf{H}^{2} \times W_{2}^{\frac{3}{2}}(\Omega) \times \widetilde{W}_{2}^{1}(\Omega) \times W_{2}^{1}(\Gamma) \text { as } \varepsilon \rightarrow+0
$$

One can easily check that $(\widetilde{\mathbf{v}}, \widetilde{T}, \widetilde{p}, \widetilde{\theta})$ is an admissible element to the problem (82-84). On the other hand, the inequality $J_{\varepsilon}\left(\widehat{\mathbf{v}}_{\varepsilon}, \widehat{T}_{\varepsilon}, \widehat{p}_{\varepsilon}, \widehat{\theta}_{\varepsilon}\right) \leq \widetilde{J}(\widehat{\mathbf{v}}, \widehat{T}, \widehat{\theta})$ implies that $(\widetilde{\mathbf{v}}, \widetilde{T}, \widetilde{p}, \widetilde{\theta})$ is the solution for the extremal problem (8284). But in this case $(\widetilde{\mathbf{v}}, \widetilde{T}, \widetilde{p}, \widetilde{\theta})=(\widehat{\mathbf{v}}, \widehat{T}, \widehat{p}, \widehat{\theta})$; otherwise

$$
\widetilde{J}(\widetilde{\mathbf{v}}, \widetilde{T}, \widetilde{\theta})+\frac{1}{2}\|\widetilde{\mathbf{v}}-\widehat{\mathbf{v}}\|_{\left[L^{2}(\Omega)\right]^{2}}^{2}+\frac{1}{2}\|\widetilde{T}-\widehat{T}\|_{L^{2}(\Omega)}^{2} \leq \widetilde{J}(\widehat{\mathbf{v}}, \widehat{T}, \widehat{\theta})
$$

But this inequality is impossible since it immediately implies that $(\hat{\mathbf{v}}, \hat{T}, \hat{p}, \hat{\theta})$ is not a solution to the problem $(82-$ 84). Hence,

$$
\left(\widehat{\mathbf{v}}_{\varepsilon}, \widehat{T}_{\varepsilon}, \widehat{p}_{\varepsilon}, \widehat{\theta}_{\varepsilon}\right) \rightarrow(\widehat{\mathbf{v}}, \widehat{T}, \widehat{p}, \widehat{\theta}) \quad \text { in } \mathbf{H}^{2} \times W_{2}^{\frac{3}{2}}(\Omega) \times \widetilde{W}_{2}^{1}(\Omega) \times W_{2}^{1}(\Gamma)
$$

To obtain the optimality system for the extremal problem (105-109), we consider the function

$$
\Phi\left(\lambda_{1}, \lambda_{2}, \lambda_{3}, \lambda_{4}\right)=J_{\varepsilon}\left(\widehat{\mathbf{v}}_{\varepsilon}+\lambda_{1} \boldsymbol{\delta}_{1}, \widehat{T}_{\varepsilon}+\lambda_{2} \delta_{2}+\lambda_{3}\left(\delta_{3}-\widehat{T}_{\varepsilon}\right), \widehat{p}_{\varepsilon}+\lambda_{4} \delta_{4}, \hat{\theta}_{\varepsilon}+\lambda_{3}\left(\theta-\widehat{\theta}_{\varepsilon}\right)\right),
$$

where $\boldsymbol{\delta}_{1} \in \mathbf{H}^{2}, \delta_{2} \in W_{2}^{2}(\Omega) \cap \stackrel{\circ}{W_{2}^{1}}(\Omega), \delta_{4} \in \widetilde{W}_{2}^{1}(\Omega)$, and the function $\delta_{3}$ is the solution to the boundary value problem

$$
\Delta \delta_{3}=0 \quad \text { in } \Omega \quad \text { and }\left.\quad \delta_{3}\right|_{\Gamma}=\theta \quad \theta \in K
$$

Obviously, this function has the global minimum at $(0,0,0,0)$ on the set $\left\{\left(\lambda_{1}, \lambda_{2}, \lambda_{3}, \lambda_{4}\right) \mid \lambda_{1}, \lambda_{2}, \lambda_{4} \in \mathbb{R}^{1}, \lambda_{3} \in\right.$ $[0,1]\}$. Applying the Fermat theorem, we obtain

$$
\left.\frac{\partial \Phi}{\partial \lambda_{i}}\right|_{(0,0,0,0)}=0, \quad i \in\{1,2,4\}, \quad \text { and }\left.\quad \frac{\partial \Phi}{\partial \lambda_{3}}\right|_{(0,0,0,0)} \geq 0 .
$$

Let

$$
R_{\varepsilon}=\frac{1}{\varepsilon} F_{2}\left(\widehat{\mathbf{v}}_{\varepsilon}, \widehat{T}_{\varepsilon}, \widehat{p}_{\varepsilon}\right) \quad \text { and } \quad \mathbf{w}_{\varepsilon}=\frac{1}{\varepsilon} \mathbf{F}_{1}\left(\widehat{\mathbf{v}}_{\varepsilon}, \widehat{T}_{\varepsilon}, \widehat{p}_{\varepsilon}\right)
$$


Obviously $R_{\varepsilon} \in L^{2}(\Omega)$ and $\mathbf{w}_{\varepsilon} \in\left[L^{2}(\Omega)\right]^{2}$. By (113), we obtain

$$
\begin{aligned}
& \left(R_{\varepsilon},-\Delta \delta_{2}+\widehat{\mathbf{v}}_{\varepsilon} \cdot \nabla \delta_{2}\right)_{L^{2}(\Omega)}+\left(\mathbf{w}_{\varepsilon},-\delta_{2} \Delta \widehat{\mathbf{v}}_{\varepsilon}-2 \nabla \delta_{2} \cdot \nabla \widehat{\mathbf{v}}_{\varepsilon}+\delta_{2} \widehat{\mathbf{v}}_{\varepsilon} \cdot \nabla \widehat{\mathbf{v}}_{\varepsilon}+\frac{\beta}{\left(1+\widehat{T}_{\varepsilon}\right)^{2}}\left(\begin{array}{c}
0 \\
1
\end{array} \delta_{2}\right)_{\left[L^{2}(\Omega)\right]^{2}}\right. \\
& +\left(\left(1+\widehat{T}_{\varepsilon}\right) \widehat{\mathbf{v}}_{\varepsilon}-\mathbf{u}_{0}, \delta_{2} \widehat{\mathbf{v}}_{\varepsilon}\right)_{\left[L^{2}(\Omega)\right]^{2}}+\left(\widehat{T}_{\varepsilon}-\widehat{T}, \delta_{2}\right)_{L^{2}(\Omega)}=0 \quad \forall \delta_{2} \in W_{2}^{2}(\Omega) \cap \stackrel{\circ}{W}_{2}^{1}(\Omega), \\
& \left(\mathbf{w}_{\varepsilon}, \nabla \delta_{4}\right)_{\left[L^{2}(\Omega)\right]^{2}}=0 \quad \forall \delta_{4} \in \widetilde{W}_{2}^{1}(\Omega), \\
& \left(R_{\varepsilon}, \boldsymbol{\delta}_{1} \cdot \nabla \widehat{T}_{\varepsilon}\right)_{L^{2}(\Omega)}+\left(\mathbf{w}_{\varepsilon},-\left(1+\widehat{T}_{\varepsilon}\right) \Delta \boldsymbol{\delta}_{1}-2 \nabla \widehat{T}_{\varepsilon} \cdot \nabla \boldsymbol{\delta}_{1}+\left(1+\widehat{T}_{\varepsilon}\right)\left(\widehat{\mathbf{v}}_{\varepsilon} \cdot \nabla \boldsymbol{\delta}_{1}+\boldsymbol{\delta}_{1} \cdot \nabla \widehat{\mathbf{v}}_{\varepsilon}\right)+q \boldsymbol{\delta}_{1}\right)_{\left[L^{2}(\Omega)\right]^{2}} \\
& +\left(\widehat{\mathbf{u}}_{\varepsilon}-\mathbf{u}_{0},\left(1+\widehat{T}_{\varepsilon}\right) \boldsymbol{\delta}_{1}\right)_{\left[L^{2}(\Omega)\right]^{2}}+\left(\widehat{\mathbf{v}}_{\varepsilon}-\widehat{\mathbf{v}}, \boldsymbol{\delta}_{1}\right)_{\left[L^{2}(\Omega)\right]^{2}}=0 \quad \forall \boldsymbol{\delta}_{1} \in \mathbf{H}^{2}, \\
& \left(R_{\varepsilon},-\Delta\left(\delta_{3}-\widehat{T}_{\varepsilon}\right)+\widehat{\mathbf{v}}_{\varepsilon} \cdot \nabla\left(\delta_{3}-\widehat{T}_{\varepsilon}\right)\right)_{L^{2}(\Omega)}+\left(\mathbf{w}_{\varepsilon},-\left(\delta_{3}-\widehat{T}_{\varepsilon}\right) \Delta \widehat{\mathbf{v}}_{\varepsilon}-2 \nabla\left(\delta_{3}-\widehat{T}_{\varepsilon}\right) \cdot \nabla \widehat{\mathbf{v}}_{\varepsilon}\right. \\
& \left.\left(\delta_{3}-\widehat{T}_{\varepsilon}\right) \widetilde{\mathbf{v}}_{\varepsilon} \cdot \nabla \widetilde{\mathbf{v}}_{\varepsilon}+\frac{\beta}{\left(1+\widehat{T}_{\varepsilon}\right)^{2}}\left(\begin{array}{c}
0 \\
1
\end{array}\right)\left(\delta_{3}-\widehat{T}_{\varepsilon}\right)\right)_{\left[L^{2}(\Omega)\right]^{2}}+\left(\left(1+\widehat{T}_{\varepsilon}\right) \widehat{\mathbf{v}}_{\varepsilon}-\mathbf{u}_{0},\left(\delta_{3}-\widehat{T}_{\varepsilon}\right) \widehat{\mathbf{v}}_{\varepsilon}\right)_{\left[L^{2}(\Omega)\right]^{2}} \\
& +\left(\widehat{T}_{\varepsilon}-\widehat{T}, \delta_{3}-\widehat{T}_{\varepsilon}\right)_{L^{2}(\Omega)}+\left(\widehat{\theta}_{\varepsilon}-\theta_{0}, \delta_{3}-\widehat{T}_{\varepsilon}\right)_{W_{2}^{1}(\Gamma)} \geq 0
\end{aligned}
$$

By (112), we can assume that $\left(\widehat{T}_{\varepsilon}, \widehat{\mathbf{v}}_{\varepsilon}\right) \in B_{r}=\left\{(T, \mathbf{v}) \mid\|(T, \mathbf{v})\|_{W_{2}^{\frac{3}{2}}(\Omega) \times \mathbf{H}^{2}} \leq r\right\}$ for all $\varepsilon \in(0,1)$ with the fixed $r$. Let

$$
\mathcal{F}_{\mu}=\left\{\delta_{2} \mid \text { there exists } z \text { such that }-\Delta \delta_{2}=z \text { in } \Omega,\left.\quad \delta_{2}\right|_{\Gamma}=0,\|z\|_{W_{2}^{-1+\mu}(\Omega)}=1\right\}
$$

Let $\mu \in(0,1)$; then, for any $\delta_{2} \in \mathcal{F}_{\mu}$, we have $\delta_{2} \in W_{2}^{1+\mu}(\Omega) \subset C^{0}(\bar{\Omega})$ and $(115)$ implies

$$
\begin{aligned}
\left\|R_{\varepsilon}\right\|_{W_{2}^{1-\mu}(\Omega)}= & \sup _{\delta_{2} \in \mathcal{F}_{\mu}}\left(R_{\varepsilon},-\Delta \delta_{2}\right)_{L^{2}(\Omega)}=\sup _{\delta_{2} \in \mathcal{F}_{\mu}}\left\{-\left(R_{\varepsilon}, \widehat{\mathbf{v}}_{\varepsilon} \cdot \nabla \delta_{2}\right)_{L^{2}(\Omega)}\right. \\
& -\left(\mathbf{w}_{\varepsilon},-\delta_{2} \Delta \widehat{\mathbf{v}}_{\varepsilon}-2 \nabla \delta_{2} \cdot \nabla \widehat{\mathbf{v}}_{\varepsilon}+\delta_{2} \widehat{\mathbf{v}}_{\varepsilon} \cdot \nabla \widehat{\mathbf{v}}_{\varepsilon}+\frac{\beta}{\left(1+\widehat{T}_{\varepsilon}\right)^{2}}\left(\begin{array}{c}
0 \\
1
\end{array}\right) \delta_{2}\right)_{\left[L^{2}(\Omega)\right]^{2}} \\
& \left.-\left(\left(1+\widehat{T}_{\varepsilon}\right) \widehat{\mathbf{v}}_{\varepsilon}-\mathbf{u}_{0}, \delta_{2} \widehat{\mathbf{v}}_{\varepsilon}\right)_{\left[L^{2}(\Omega)\right]^{2}}-\left(\widehat{T}_{\varepsilon}-\widehat{T}, \delta_{2}\right)_{L^{2}(\Omega)}\right\} \\
& \leq C_{1}(\mu, r)\left(1+\left\|R_{\varepsilon}\right\|_{L^{2}(\Omega)}+\left\|\mathbf{w}_{\varepsilon}\right\|_{\left[L^{2}(\Omega)\right]^{2}}+\left\|\widehat{T}_{\varepsilon}-\widehat{T}\right\|_{L^{2}(\Omega)}\right)
\end{aligned}
$$


Let

$$
\begin{aligned}
\mathcal{G}_{\mu}^{\ell}=\left\{\boldsymbol{\delta}_{1} \in\left[L^{2}(\Omega)\right]^{2} \mid \text { there exist }(\mathbf{z}, \sigma)\right. \text { such that } \\
\\
\left.\quad-\Delta \boldsymbol{\delta}_{1}=\nabla \sigma+\mathbf{z},\left.\quad \boldsymbol{\delta}_{1}\right|_{\Gamma}=\mathbf{0}, \quad \nabla \cdot \boldsymbol{\delta}_{1}=0,\|\mathbf{z}\|_{\left[W_{\ell}^{-1+\mu}(\Omega)\right]^{2}}=1\right\} .
\end{aligned}
$$

Then, from (117-119) and the Sobolev embedding theorem, we have

$$
\begin{aligned}
\left\|\mathbf{w}_{\varepsilon}\right\|_{\left[L^{4}(\Omega)\right]^{2}} \leq & C_{2}\left\|\mathbf{w}_{\varepsilon}\right\|_{\left[W_{\frac{4}{3}}^{1}(\Omega)\right]^{2}} \\
\leq & C(r)\left(\left\|\left(1+\widehat{T}_{\varepsilon}\right) \mathbf{w}_{\varepsilon}\right\|_{\left[W_{\frac{4}{3}}^{1}(\Omega)\right]^{2}}+\left\|\mathbf{w}_{\varepsilon}\right\|_{\left[L^{2}(\Omega)\right]^{2}}\right) \\
\leq & C_{3}(r)\left(\sup _{\boldsymbol{\delta}_{1} \in \mathcal{G}_{0}^{4}}\left(\mathbf{w}_{\varepsilon},-\left(1+\widehat{T}_{\varepsilon}\right) \Delta \boldsymbol{\delta}_{1}\right)_{\left[L^{2}(\Omega)\right]^{2}}+\left\|\mathbf{w}_{\varepsilon}\right\|_{\left[L^{2}(\Omega)\right]^{2}}\right) \\
\leq & C_{4}(r)\left(\operatorname { s u p } _ { \boldsymbol { \delta } _ { 1 } \in \mathcal { G } _ { 0 } ^ { 4 } } \left\{\left(\mathbf{w}_{\varepsilon}, 2 \nabla \widehat{T}_{\varepsilon} \cdot \nabla \boldsymbol{\delta}_{1}-\left(1+\widehat{T}_{\varepsilon}\right)\left(\widehat{\mathbf{v}}_{\varepsilon} \cdot \nabla \boldsymbol{\delta}_{1}+\boldsymbol{\delta}_{1} \cdot \nabla \widehat{\mathbf{v}}_{\varepsilon}\right)-q \boldsymbol{\delta}_{1}\right)_{\left[L^{2}(\Omega)\right]^{2}}\right.\right. \\
& -\left(\widehat{\mathbf{u}}_{\varepsilon}-\mathbf{u}_{0},\left(1+\widehat{T}_{\varepsilon}\right) \boldsymbol{\delta}_{1}\right)_{\left[L^{2}(\Omega)\right]^{2}}-\left(\widehat{\mathbf{v}}_{\varepsilon}-\widehat{\mathbf{v}}, \boldsymbol{\delta}_{1}\right)_{\left[L^{2}(\Omega)\right]^{2}} \\
& \left.\left.-\left(R_{\varepsilon}, \boldsymbol{\delta}_{1} \cdot \nabla \widehat{T}_{\varepsilon}\right)_{L^{2}(\Omega)}\right\}+\left\|\mathbf{w}_{\varepsilon}\right\|_{\left(L^{2}(\Omega)\right)^{2}}\right) \\
\leq & C_{5}(r)\left(1+\left\|R_{\varepsilon}\right\|_{L^{2}(\Omega)}+\left\|\mathbf{w}_{\varepsilon}\right\|_{\left[L^{2}(\Omega)\right]^{2}}+\left\|\widehat{T}_{\varepsilon}-\widehat{T}\right\|_{L^{2}(\Omega)}+\left\|\widehat{\mathbf{v}}_{\varepsilon}-\widehat{\mathbf{v}}\right\|_{\left[L^{2}(\Omega)\right]^{2}}\right) .
\end{aligned}
$$

Moreover,

$$
\begin{aligned}
\left\|\mathbf{w}_{\varepsilon}\right\|_{\left[W_{4}^{1}(\Omega)\right]^{2} \leq} & C_{6}(r)\left(\left\|\left(1+\widehat{T}_{\varepsilon}\right) \mathbf{w}_{\varepsilon}\right\|_{\left[W_{4}^{1}(\Omega)\right]^{2}}+\left\|\mathbf{w}_{\varepsilon}\right\|_{\left[L^{2}(\Omega)\right]^{2}}\right) \\
\leq & C_{7}(r)\left(\sup _{\boldsymbol{\delta}_{1 \in \mathcal{G}_{0}^{\frac{4}{3}}}}\left(\mathbf{w}_{\varepsilon},-\left(1+\widehat{T}_{\varepsilon}\right) \Delta \boldsymbol{\delta}_{1}\right)_{L^{2}(\Omega)}+\left\|\mathbf{w}_{\varepsilon}\right\|_{\left[L^{2}(\Omega)\right]^{2}}\right) \\
\leq & C_{8}(r)\left(\operatorname { s u p } _ { \boldsymbol { \delta } _ { 1 } \in \mathcal { G } _ { 0 } ^ { \frac { 4 } { 3 } } } \left\{\left(\mathbf{w}_{\varepsilon}, 2 \nabla \widehat{T}_{\varepsilon} \cdot \nabla \boldsymbol{\delta}_{1}-\left(1+\widehat{T}_{\varepsilon}\right)\left(\widehat{\mathbf{v}}_{\varepsilon} \cdot \nabla \boldsymbol{\delta}_{1}+\boldsymbol{\delta}_{1} \cdot \nabla \widehat{\mathbf{v}}_{\varepsilon}\right)-q \boldsymbol{\delta}_{1}\right)_{\left[L^{2}(\Omega)\right]^{2}}\right.\right. \\
& -\left(\widehat{\mathbf{u}}_{\varepsilon}-\mathbf{u}_{0},\left(1+\widehat{T}_{\varepsilon}\right) \boldsymbol{\delta}_{1}\right)_{\left[L^{2}(\Omega)\right]^{2}}-\left(\widehat{\mathbf{v}}_{\varepsilon}-\widehat{\mathbf{v}}, \boldsymbol{\delta}_{1}\right)_{\left[L^{2}(\Omega)\right]^{2}} \\
& \left.\left.-\left(R_{\varepsilon}, \boldsymbol{\delta}_{1} \cdot \nabla \widehat{T}_{\varepsilon}\right)_{L^{2}(\Omega)}\right\}+\left\|\mathbf{w}_{\varepsilon}\right\|_{\left(L^{2}(\Omega)\right)^{2}}\right) \\
\leq & C_{9}(r)\left(1+\left\|R_{\varepsilon}\right\|_{L^{2}(\Omega)}+\left\|\mathbf{w}_{\varepsilon}\right\|_{[C(\Omega)]^{2}}+\left\|\widehat{T}_{\varepsilon}-\widehat{T}\right\|_{L^{2}(\Omega)}+\left\|\widehat{\mathbf{v}}_{\varepsilon}-\widehat{\mathbf{v}}\right\|_{\left[L^{2}(\Omega)\right]^{2}}\right) .
\end{aligned}
$$

The inequalities (119-121) yield

$$
\left\|\mathbf{w}_{\varepsilon}\right\|_{\left[W_{4}^{1}(\Omega)\right]^{2}} \leq C_{10}(r)\left(1+\left\|R_{\varepsilon}\right\|_{L^{2}(\Omega)}+\left\|\mathbf{w}_{\varepsilon}\right\|_{\left[L^{2}(\Omega)\right]^{2}}+\left\|\widehat{T}_{\varepsilon}-\widehat{T}\right\|_{L^{2}(\Omega)}+\left\|\widehat{\mathbf{v}}_{\varepsilon}-\widehat{\mathbf{v}}\right\|_{\left[L^{2}(\Omega)\right]^{2}}\right) .
$$


On the other hand, the improved regularity for $\mathbf{w}_{\varepsilon}$ and (115) imply

$$
\begin{aligned}
\left\|R_{\varepsilon}\right\|_{W_{2}^{1}(\Omega)}= & \sup _{\delta_{2} \in \mathcal{F}_{0}}\left(R_{\varepsilon},-\Delta \delta_{2}\right)_{L^{2}(\Omega)}=\sup _{\delta_{2} \in \mathcal{F}_{0}}\left\{-\left(R_{\varepsilon}, \widehat{\mathbf{v}}_{\varepsilon} \cdot \nabla \delta_{2}\right)_{L^{2}(\Omega)}\right. \\
& -\left(\mathbf{w}_{\varepsilon},-\delta_{2} \Delta \widehat{\mathbf{v}}_{\varepsilon}-2 \nabla \delta_{2} \cdot \nabla \widehat{\mathbf{v}}_{\varepsilon}+\delta_{2} \widehat{\mathbf{v}}_{\varepsilon} \cdot \nabla \widehat{\mathbf{v}}_{\varepsilon}+\frac{\beta}{\left(1+\widehat{T}_{\varepsilon}\right)^{2}}\left(\begin{array}{c}
0 \\
1
\end{array}\right) \delta_{2}\right)_{\left[L^{2}(\Omega)\right]^{2}} \\
& \left.-\left(\left(1+\widehat{T}_{\varepsilon}\right) \widehat{\mathbf{v}}_{\varepsilon}-\mathbf{u}_{0}, \delta_{2} \widehat{\mathbf{v}}_{\varepsilon}\right)_{\left[L^{2}(\Omega)\right]^{2}}-\left(\widehat{T}_{\varepsilon}-\widehat{T}_{2}, \delta_{2}\right)_{L^{2}(\Omega)}\right\} \\
\leq & C_{11}(r)\left(1+\left\|R_{\varepsilon}\right\|_{L^{2}(\Omega)}+\left\|\mathbf{w}_{\varepsilon}\right\|_{\left[L^{2}(\Omega)\right]^{2}}+\left\|\widehat{T}_{\varepsilon}-\widehat{T}\right\|_{L^{2}(\Omega)}\right)
\end{aligned}
$$

Equations (115-118) along with (122) and (123) imply

$$
\begin{gathered}
-\Delta\left(\left(1+\widehat{T}_{\varepsilon}\right) \mathbf{w}_{\varepsilon}\right)-\widehat{\mathbf{v}}_{\varepsilon} \cdot \nabla\left(\left(1+\widehat{T}_{\varepsilon}\right) \mathbf{w}_{\varepsilon}\right)+\left(1+\widehat{T}_{\varepsilon}\right) \mathbf{w}_{\varepsilon} \cdot\left(\nabla \widehat{\mathbf{v}}_{\varepsilon}\right)^{T}+q \mathbf{w}_{\varepsilon}+2 \nabla \widehat{T}_{\varepsilon} \cdot \nabla \mathbf{w}_{\varepsilon} \\
+R_{\varepsilon} \nabla \widehat{T}_{\varepsilon}+2 \mathbf{w}_{\varepsilon} \Delta \widehat{T}_{\varepsilon}+\nabla \sigma_{\varepsilon}+\left(\widehat{\mathbf{u}}_{\varepsilon}-\mathbf{u}_{0}\right)\left(1+\widehat{T}_{\varepsilon}\right)+\widehat{\mathbf{v}}_{\varepsilon}-\widehat{\mathbf{v}}=\mathbf{0} \quad \text { in } \Omega \\
-\Delta R_{\varepsilon}-\widehat{\mathbf{v}}_{\varepsilon} \cdot \nabla R_{\varepsilon}+\frac{\beta}{\left(1+\widehat{T}_{\varepsilon}\right)^{2}}\left(\begin{array}{c}
0 \\
1
\end{array}\right) \cdot \mathbf{w}_{\varepsilon}-\mathbf{w}_{\varepsilon} \cdot \Delta \widehat{\mathbf{v}}_{\varepsilon}+2 \nabla \mathbf{w}_{\varepsilon}: \nabla \widehat{\mathbf{v}}_{\varepsilon} \\
+R_{\varepsilon}\left(\widehat{\mathbf{v}}_{\varepsilon} \cdot \nabla \widehat{\mathbf{v}}_{\varepsilon}\right) \cdot \mathbf{w}_{\varepsilon}+\left(\widehat{\mathbf{u}}_{\varepsilon}-\mathbf{u}_{0}\right) \cdot \widehat{\mathbf{v}}_{\varepsilon}+\left(\widehat{T}_{\varepsilon}-\widehat{T}\right)=0 \quad \text { in } \Omega, \\
\nabla \cdot \mathbf{w}_{\varepsilon}=0 \quad \text { in } \Omega \\
\left.\mathbf{w}_{\varepsilon}\right|_{\Gamma}=\mathbf{0} \\
\left.R_{\varepsilon}\right|_{\Gamma}=0
\end{gathered}
$$

and

$$
\left(-\Delta_{\Gamma}\left(\widehat{\theta}_{\varepsilon}-\theta_{0}\right)+\lambda\left(\widehat{\theta}_{\varepsilon}-\theta_{0}\right)+\frac{\partial R_{\varepsilon}}{\partial \nu}, \theta-\widehat{\theta}_{\varepsilon}\right)_{L^{2}(\Gamma)} \geq 0 \quad \forall \theta \in K
$$

By (122) and (123), we obtain from (124)

$$
\left\|\sigma_{\varepsilon}\right\|_{L^{4}(\Omega)} \leq C_{12}(r)\left(1+\left\|R_{\varepsilon}\right\|_{L^{2}(\Omega)}+\left\|\mathbf{w}_{\varepsilon}\right\|_{\left[L^{2}(\Omega)\right]^{2}}+\left\|\widehat{\mathbf{v}}_{\varepsilon}-\widehat{\mathbf{v}}\right\|_{\left[L^{2}(\Omega)\right]^{2}}\right) .
$$

Moreover $(122,124)$, and a priori estimates for elliptic boundary value problems applied to (125) and (128) imply

$$
\left\|R_{\varepsilon}\right\|_{W_{2}^{2}(\Omega)} \leq C_{13}(r)\left(1+\left\|R_{\varepsilon}\right\|_{L^{2}(\Omega)}+\left\|\mathbf{w}_{\varepsilon}\right\|_{\left[L^{2}(\Omega)\right]^{2}}+\left\|\widehat{T}_{\varepsilon}-\widehat{T}\right\|_{L^{2}(\Omega)}\right) .
$$


Using (4), one can rewrite (124) as

$$
-\Delta \mathbf{w}_{\varepsilon}+\nabla\left(\widehat{\rho}_{\varepsilon} \sigma_{\varepsilon}\right)=\mathbf{m}_{\varepsilon} \quad \text { in } \Omega, \quad \nabla \cdot \mathbf{w}_{\varepsilon}=0 \quad \text { in } \Omega, \quad \text { and }\left.\quad \mathbf{w}_{\varepsilon}\right|_{\Gamma}=\mathbf{0},
$$

where

$$
\begin{aligned}
\mathbf{m}_{\varepsilon}= & \hat{\rho}_{\varepsilon}\left(-\sigma_{\varepsilon} \widehat{\rho}_{\varepsilon} \nabla \widehat{T}_{\varepsilon}+\widehat{\mathbf{v}}_{\varepsilon} \cdot \nabla\left(1+T_{\varepsilon}\right) \mathbf{w}_{\varepsilon}-\left(1+\hat{T}_{\varepsilon}\right) \mathbf{w}_{\varepsilon} \cdot\left(\nabla \widehat{\mathbf{v}}_{\varepsilon}\right)^{T}-q \mathbf{w}_{\varepsilon}\right. \\
& \left.-R_{\varepsilon} \nabla \hat{T}_{\varepsilon}+\Delta \widehat{T}_{\varepsilon} \mathbf{w}_{\varepsilon}-\left(\widehat{\mathbf{u}}_{\varepsilon}-\mathbf{u}_{0}\right)\left(1+\widehat{T}_{\varepsilon}\right)-\widehat{\mathbf{v}}_{\varepsilon}+\widehat{\mathbf{v}}\right)
\end{aligned}
$$

where $\widehat{\rho}_{\varepsilon}\left(1+\widehat{T}_{\varepsilon}\right)=1$ in $\Omega$.

By $(122,130)$, and $(131)$, we have

$$
\left\|\mathbf{m}_{\varepsilon}\right\|_{\left[L^{2}(\Omega)\right]^{2}} \leq C_{14}(r)\left(1+\left\|R_{\varepsilon}\right\|_{L^{2}(\Omega)}+\left\|\mathbf{w}_{\varepsilon}\right\|_{\left[L^{2}(\Omega)\right]^{2}}+\left\|\widehat{\mathbf{v}}_{\varepsilon}-\widehat{\mathbf{v}}\right\|_{\left[L^{2}(\Omega)\right]^{2}}\right) .
$$

This inequality and standard a priori estimates for the Stokes system imply

$$
\left\|\sigma_{\varepsilon}\right\|_{\widetilde{W}_{2}^{1}(\Omega)}+\left\|\mathbf{w}_{\varepsilon}\right\|_{\mathbf{H}^{2}} \leq C_{15}(r)\left(1+\left\|R_{\varepsilon}\right\|_{L^{2}(\Omega)}+\left\|\mathbf{w}_{\varepsilon}\right\|_{\left[L^{2}(\Omega)\right]^{2}}+\left\|\widehat{\mathbf{v}}_{\varepsilon}-\widehat{\mathbf{v}}\right\|_{\left[L^{2}(\Omega)\right]^{2}}+\left\|\widehat{T}_{\varepsilon}-\widehat{T}\right\|_{L^{2}(\Omega)}\right) .
$$

Let $I_{\varepsilon}=\left\|R_{\varepsilon}\right\|_{L^{2}(\Omega)}+\left\|\mathbf{w}_{\varepsilon}\right\|_{\left[L^{2}(\Omega)\right]^{2}}+\left\|\sigma_{\varepsilon}\right\|_{L^{2}(\Omega)}$. We consider two cases. First, assume that $\underline{\lim }_{\varepsilon \rightarrow 0} I_{\varepsilon}<\infty$. Then, by (131) and (132), the sequence

$$
\left\{\left(R_{\varepsilon}, \mathbf{w}_{\varepsilon}, \sigma_{\varepsilon}\right)\right\} \text { is bounded in } W_{2}^{2}(\Omega) \times \mathbf{H}^{2} \times \widetilde{W}_{2}^{1}(\Omega) .
$$

Hence, taking a subsequence if necessary, we have that

$$
\left(R_{\varepsilon}, \mathbf{w}_{\varepsilon}, \sigma_{\varepsilon}\right) \rightarrow(R, \mathbf{w}, \sigma) \quad \text { weakly in } W_{2}^{2}(\Omega) \times \mathbf{H}^{2} \times \widetilde{W}_{2}^{1}(\Omega) \text { as } \varepsilon \rightarrow+0 .
$$

Passing to the limit as $\varepsilon \rightarrow+0$ in (124-128), using $(112,134)$, we obtain that $(R, \mathbf{w}, \sigma)$ satisfies (98-103) with $\lambda=-1$. To pass to the limit in (129), first note that

$$
\begin{aligned}
\left(-\Delta_{\Gamma}\left(\widehat{\theta}_{\varepsilon}-\theta_{0}\right)+\left(\widehat{\theta}_{\varepsilon}-\theta_{0}\right), \theta-\widehat{\theta}_{\varepsilon}\right)_{L^{2}(\Gamma)} & =\left(\widehat{\theta}_{\varepsilon}-\theta_{0}, \theta-\widehat{\theta}_{\varepsilon}\right)_{W_{2}^{1}(\Gamma)}= \\
& -\left\|\widehat{\theta}_{\varepsilon}\right\|_{W_{2}^{1}(\Gamma)}^{2}+\left(\theta_{0}, \widehat{\theta}_{\varepsilon}\right)_{W_{2}^{1}(\Gamma)}-\left(\theta_{0}, \widehat{\theta}\right)_{W_{2}^{1}(\Gamma)}+\left(\theta, \widehat{\theta}_{\varepsilon}\right)_{W_{2}^{1}(\Gamma)} .
\end{aligned}
$$

By (112), passing to the limit in (135) as $\varepsilon \rightarrow+0$, we obtain

$$
\begin{aligned}
\lim _{\varepsilon \rightarrow+0}\left(-\Delta_{\Gamma}\left(\widehat{\theta}_{\varepsilon}-\theta_{0}\right)+\widehat{\theta}_{\varepsilon}-\theta_{0}, \theta-\widehat{\theta}_{\varepsilon}\right)_{L^{2}(\Omega)} & =\lim _{\varepsilon \rightarrow+0}\left(\widehat{\theta}_{\varepsilon}-\theta_{0}, \theta-\widehat{\theta}_{\varepsilon}\right)_{W_{2}^{1}(\Gamma)} \\
& =-\|\widehat{\theta}\|_{W_{2}^{1}(\Gamma)}^{2}+\left(\theta_{0}, \widehat{\theta}\right)_{W_{2}^{1}(\Gamma)}-\left(\theta_{0}, \theta\right)_{W_{2}^{1}(\Gamma)}+(\theta, \widehat{\theta})_{W_{2}^{1}(\Gamma)} .
\end{aligned}
$$

Moreover, by (134) and the trace theorem,

$$
\widehat{\theta}_{\varepsilon} \rightarrow \hat{\theta} \text { in } W_{2}^{1}(\Gamma) \quad \text { and } \quad \frac{\partial R_{\varepsilon}}{\partial \nu} \rightarrow \frac{\partial R}{\partial \nu} \quad \text { weakly in } W_{2}^{\frac{1}{2}}(\Gamma) .
$$

This convergence implies immediately

$$
\lim _{\varepsilon \rightarrow+0}\left(\frac{\partial R_{\varepsilon}}{\partial \nu}, \theta-\widehat{\theta}_{\varepsilon}\right)_{L^{2}(\Gamma)}=\left(\frac{\partial R}{\partial \nu}, \theta-\widehat{\theta}\right)_{L^{2}(\Gamma)} .
$$


Finally, passing to the limit in (129), we obtain that the pair $(R, \widehat{\theta})$ satisfies (104).

For the second case, assume that

$$
\varliminf_{\varepsilon \rightarrow+0} I_{\varepsilon}=+\infty .
$$

Let us introduce the new variables

$$
\widetilde{R}_{\varepsilon}=\frac{R_{\varepsilon}}{I_{\varepsilon}}, \quad \widetilde{\mathbf{w}}_{\varepsilon}=\frac{\mathbf{w}_{\varepsilon}}{I_{\varepsilon}}, \quad \text { and } \quad \widetilde{\sigma}_{\varepsilon}=\frac{\sigma_{\varepsilon}}{I_{\varepsilon}} .
$$

By (124-129), the triple $\left(\widetilde{R}_{\varepsilon}, \widetilde{\mathbf{w}}_{\varepsilon}, \sigma_{\varepsilon}\right)$ satisfies to the system of equations

$$
\begin{gathered}
-\Delta\left(1+\widehat{T}_{\varepsilon}\right) \widetilde{\mathbf{w}}_{\varepsilon}-\widehat{\mathbf{v}}_{\varepsilon} \cdot \nabla\left(\left(1+\hat{T}_{\varepsilon}\right) \widetilde{\mathbf{w}}_{\varepsilon}\right)+\left(1+\hat{T}_{\varepsilon}\right) \widetilde{\mathbf{w}}_{\varepsilon} \cdot\left(\widehat{\mathbf{v}}_{\varepsilon}\right)^{T}+q \widetilde{\mathbf{w}}_{\varepsilon}+\widetilde{R}_{\varepsilon} \nabla \hat{T}_{\varepsilon} \\
+2 \nabla \widehat{T}_{\varepsilon} \cdot \nabla \widetilde{\mathbf{w}}_{\varepsilon}+2 \Delta \widehat{T}_{\varepsilon} \widetilde{\mathbf{w}}_{\varepsilon}+\nabla \widetilde{\sigma}_{\varepsilon}+\left\{\left(\widehat{\mathbf{u}}_{\varepsilon}-\mathbf{u}_{0}\right)\left(1+\widehat{T}_{\varepsilon}\right)+\widehat{\mathbf{v}}_{\varepsilon}-\widehat{\mathbf{v}}\right\} / I_{\varepsilon}=\mathbf{0} \quad \text { in } \Omega, \\
-\Delta \widetilde{R}_{\varepsilon}-\widehat{\mathbf{v}}_{\varepsilon} \cdot \nabla \widetilde{R}_{\varepsilon}+\frac{\beta}{\left(1+\widehat{T}_{\varepsilon}\right)^{2}}\left(\begin{array}{c}
0 \\
1
\end{array}\right) \cdot \widetilde{\mathbf{w}}_{\varepsilon}-\widetilde{\mathbf{w}}_{\varepsilon} \cdot \Delta \widehat{\mathbf{v}}_{\varepsilon}+2 \nabla \widetilde{\mathbf{w}}_{\varepsilon}: \nabla \widehat{\mathbf{v}}_{\varepsilon} \\
+\widetilde{R}_{\varepsilon}\left(\widehat{\mathbf{v}}_{\varepsilon} \cdot \nabla \widehat{\mathbf{v}}_{\varepsilon}\right) \cdot \widetilde{\mathbf{w}}_{\varepsilon}+\left\{\left(\widehat{\mathbf{u}}_{\varepsilon}-\mathbf{u}_{0}\right) \cdot \widehat{\mathbf{v}}_{\varepsilon}+\left(\widehat{T}_{\varepsilon}-\widehat{T}\right)\right\} / I_{\varepsilon}=0 \text { in } \Omega, \\
\nabla \cdot \widetilde{\mathbf{w}}_{\varepsilon}=0 \quad \text { in } \Omega \\
\left.\widetilde{\mathbf{w}}_{\varepsilon}\right|_{\Gamma}=\mathbf{0} \\
\left.\widetilde{R}_{\varepsilon}\right|_{\Gamma}=0
\end{gathered}
$$

and

$$
\left(\left(-\Delta_{\Gamma}\left(\widehat{\theta}_{\varepsilon}-\theta_{0}\right)+\left(\widehat{\theta}_{\varepsilon}-\theta_{0}\right)\right) / I_{\varepsilon}+\frac{\partial \widetilde{R}_{\varepsilon}}{\partial \nu}, \theta_{\varepsilon}-\widehat{\theta}_{\varepsilon}\right)_{L^{2}(\Gamma)} \geq 0 \quad \forall \theta \in K .
$$

Note that by (112) and (137), we have

$$
\left\{\left(\widehat{\mathbf{u}}_{\varepsilon}-\mathbf{u}_{0}\right) \cdot \widehat{\mathbf{v}}_{\varepsilon}+\left(\widehat{T}_{\varepsilon}-\widehat{T}\right)\right\} / I_{\varepsilon} \rightarrow 0 \quad \text { in } L^{2}(\Omega)
$$

and

$$
\left\{\left(\widehat{\mathbf{u}}_{\varepsilon}-\mathbf{u}_{0}\right)\left(1+\widehat{T}_{\varepsilon}\right)+\left(\widehat{\mathbf{v}}_{\varepsilon}-\widehat{\mathbf{v}}\right)\right\} / I_{\varepsilon} \rightarrow 0 \quad \text { in }\left[L^{2}(\Omega)\right]^{2} .
$$

By (144) and (145), using the arguments similar to (120-131), we have

$$
\left\{\left(\widetilde{R}_{\varepsilon}, \widetilde{\mathbf{w}}_{\varepsilon}, \widetilde{\sigma}_{\varepsilon}\right)\right\}_{\{\varepsilon \in(0,1)\}} \text { is bounded in } W_{2}^{2}(\Omega) \times \mathbf{H}^{2} \times \widetilde{W}_{2}^{1}(\Omega) .
$$


So, keeping in mind that

$$
\left\|\left(\widetilde{R}_{\varepsilon}, \widetilde{\mathbf{w}}_{\varepsilon}, \widetilde{\sigma}_{\varepsilon}\right)\right\|_{L^{2}(\Omega) \times\left[L^{2}(\Omega)\right]^{2} \times \widetilde{L}^{2}(\Omega)}=1
$$

and taking if necessary a subsequence, we obtain

$$
\left(\widetilde{R}_{\varepsilon}, \widetilde{\mathbf{w}}_{\varepsilon}, \widetilde{\sigma}_{\varepsilon}\right) \rightarrow(R, \mathbf{w}, \sigma) \quad \text { weakly in } W_{2}^{2}(\Omega) \times \mathbf{H}^{2} \times \widetilde{W}_{2}^{1}(\Omega) .
$$

Moreover $(R, \mathbf{w}, \sigma) \neq 0$. Passing to the limit in (138-143) in a similar manner to the first case, we obtain that triple the $(R, \mathbf{w}, \sigma)$ satisfies (97-104) with $\lambda=0$. Also, if $R \equiv 0$ and $\mathbf{w} \equiv 0$, then by (98), $\nabla \sigma \equiv 0$. Hence, by the definition of the space $\widetilde{L}^{2}(\Omega)$, we have that $\sigma \equiv 0$. But this fact contradicts (146). Thus, the proof is complete.

Remark. The system (1-6) is a special case of the general system for which (1) is replaced by

$$
-\nu \Delta \mathbf{u}+\rho \mathbf{u} \cdot \nabla \mathbf{u}+\nabla p+\beta\left(\begin{array}{c}
0 \\
1
\end{array}\right) \rho T=\mathbf{f} \quad \text { in } \Omega .
$$

\section{REFERENCES}

[1] F. Durst, A. Galjukov, Y. Makarov, M. Shafer, P. Voinovich and A. Zhmakin, Efficient 3D unstructured grid algorithms for modelling of chemical vapour deposition in horizontal reactors, in Simulation of Semiconductor Devices and Processes, Vol. 6, edited by H. Ryssel and P. Pichler (1995) 258-261.

[2] N. Efimov and S. Stechkin, Approximative compactness and Tchebycheff sets. Soviet Math. 2 (1961) 1226-1228.

[3] E. Einset and K. Jensen, Finite element solution of three-dimensional mixed convection gas flows in horizontal channels using preconditioned iterative methods. Int. J. Numer. Meth. Engrg. 14 (1992) 817-841.

[4] C. Forester and A. Emery, A computational method for low Mach number unsteady compressible free convective flows. J. Comput. Phys. 10 (1972) 487-502.

[5] J.-L. Lions and E. Magenes, Non-homogeneous Boundary Value Problems and Applications 1. Springer, New York (1972).

[6] Y. Makarov and A. Zhmakin, On the flow regimes in VPE reactors. J. Cryst. Growth 94 (1989) 537-550.

[7] J. Serrin, Mathematical priciples of classical fluid mechanics, in Handbuch der Physik VIII/1, edited by S. Flügge and C. Truesdell. Springer (1959) 1-125.

[8] R. Temam, Navier-Stokes Equations. North-Holland, Amsterdam (1979).

[9] L. Vlasov, Approximate properties of sets in normed linear spaces. Russian Math. Surveys 28 (1973) 1-66. 